\title{
Winner's curse correction and variable thresholding improve performance of polygenic risk modeling based on genome-wide association study summary-level data
}

Jianxin Shi ${ }^{1}$, Ju-Hyun Park ${ }^{2}$, Jubao Duan ${ }^{3}$, Sonja Berndt ${ }^{1}$, Winton Moy ${ }^{4}$, Kai Yu ${ }^{1}$, Lei Song ${ }^{1}$, William Wheeler $^{5}$, Xing Hua ${ }^{1}$, Debra Silverman ${ }^{1}$, Montserrat Garcia-Closas ${ }^{1}$, Chao Agnes Hsiung ${ }^{6}$, Jonine D Figueroa $^{1,7}$, Victoria K Cortessis ${ }^{8,9}$, Núria Malats ${ }^{10}$, Margaret R Karagas ${ }^{11}$, Paolo Vineis ${ }^{12,13}$, I-Shou Chang $^{14}$, Dongxin Lin ${ }^{15,16}$, Baosen Zhou ${ }^{17}$, Adeline Seow ${ }^{18}$, Keitaro Matsuo ${ }^{19}$, Yun-Chul Hong ${ }^{20}$, Neil E. Caporaso ${ }^{1}$, Brian Wolpin ${ }^{21,22}$, Eric Jacobs ${ }^{23}$, Gloria Petersen ${ }^{24}$, Alison P. Klein ${ }^{25,26}$, Donghui Li $^{27}$, Harvey Risch $^{28}$, Alan R. Sanders ${ }^{3}$, Li Hsu ${ }^{29}$, Robert E. Schoen ${ }^{30}$, Hermann Brenner ${ }^{31,32,33}$, MGS (Molecular Genetics of Schizophrenia) GWAS Consortium, GECCO (The Genetics and Epidemiology of Colorectal Cancer Consortium), The GAME-ON/TRICL (Transdisciplinary Research in Cancer of the Lung) GWAS Consortium, PRACTICAL (PRostate cancer AssoCiation group To Investigate Cancer Associated aLterations) Consortium, PanScan and PanC4 Consortium, The GAMEON/ELLIPSE Consortium, Rachael Stolzenberg-Solomon ${ }^{1}$, Pablo Gejman ${ }^{3}$, Qing Lan ${ }^{1}$, Nathaniel Rothman ${ }^{1}$, Laufey T. Amundadottir ${ }^{1}$, Maria Teresa Landi ${ }^{1}$, Douglas F. Levinson ${ }^{34}$, Stephen J. Chanock $^{1}$, Nilanjan Chatterjee ${ }^{1,35}$.

${ }^{1}$ Division of Cancer Epidemiology and Genetics, National Cancer Institute, Bethesda, Mayland 20892, USA. ${ }^{2}$ Statistics Department, Dongguk University, Seoul, Korea. ${ }^{3}$ Center for Psychiatric Genetics, Department of Psychiatry and Behavioral Sciences, North Shore University Health System Research Institute, University of Chicago Pritzker School of Medicine, Evanston, Illinois 60201, USA. ${ }^{4}$ Dept. of Statistics, Northern Illinois Unviersity, DeKalb, IL, 60115, USA. ${ }^{5}$ Information Management Services, Inc., Rockville, MD, 20852, USA. ${ }^{6}$ Institute of Population Health Sciences, National Health Research Institutes, Miaoli, Taiwan. ${ }^{7}$ Usher Institute of Population Health Sciences and Informatics, The University of Edinburgh, Medical School, Edinburgh, United Kingdom. ${ }^{8}$ Department of Preventive Medicine and Department of Obstetrics and Gynecology, USC Keck School of Medicine, University of Southern California, Los Angeles, California 90033, USA. ${ }^{9}$ Norris Comprehensive Cancer Center, USC Keck School of Medicine, University of Southern California, Los Angeles, California 90033, USA. ${ }^{10}$ Genetic and Molecular Epidemiology Group, Spanish National Cancer Research Centre (CNIO), Madrid, Spain. ${ }^{11}$ Geisel School of Medicine, Dartmouth College, Hanover, New Hampshire, USA. ${ }^{12}$ Human Genetics Foundation, Turin, Italy. ${ }^{13}$ MRC-PHE Centre for Environment and Health, School of Public Health, Imperial College London, London, United Kingdom. ${ }^{14}$ National Institute of Cancer Research, National Health Research Institutes, Zhunan, Taiwan. ${ }^{15}$ Department of Etiology \& Carcinogenesis, Cancer Institute and Hospital, Chinese Academy of Medical Sciences and Peking Union Medical College, Beijing, China. ${ }^{16}$ State Key Laboratory of Molecular Oncology, Cancer Institute and Hospital, Chinese Academy of Medical Sciences and Peking Union Medical College, Beijing, China. ${ }^{17}$ Department of Epidemiology, School of Public Health, China Medical University, Shenyang, China. ${ }^{18}$ Saw Swee Hock School of Public Health, National University of Singapore, Singapore. ${ }^{19}$ Division of Molecular Medicine, Aichi Cancer Center Research Institute, 1-1 Kanokoden, Chikusa-ku, Nagoya 464-8681, Japan. ${ }^{20}$ Department of Preventive Medicine, Seoul National 
University College of Medicine, Seoul, Republic of Korea. ${ }^{21}$ Department of Medical Oncology, DanaFarber Cancer Institute, Boston, Massachusetts, USA. ${ }^{22}$ Department of Medicine, Brigham and Women's Hospital and Harvard Medical School, Boston, Massachusetts, USA. ${ }^{23}$ Epidemiology Research Program, American Cancer Society, Atlanta, Georgia, USA. ${ }^{24}$ Division of Epidemiology, Department of Health Sciences Research, Mayo Clinic, Rochester, Minnesota, USA. ${ }^{25}$ Department of Oncology, the Johns Hopkins University School of Medicine, Baltimore, Maryland, USA.

${ }^{26}$ Department of Epidemiology, the Bloomberg School of Public Health, Baltimore, Maryland, USA.

${ }^{27}$ Department of Gastrointestinal Medical Oncology, University of Texas M.D. Anderson Cancer Center, Houston, Texas, USA. ${ }^{28}$ Department of Chronic Disease Epidemiology, Yale School of Public Health, New Haven, Connecticut, USA. ${ }^{29}$ Public Health Sciences Division, Fred Hutchinson Cancer Research Center, Seattle, WA 98109, USA. ${ }^{30}$ Department of Medicine and Epidemiology, University of Pittsburgh Medical Center, Pittsburgh, PA 15260, USA. ${ }^{31}$ Division of Clinical Epidemiology and Aging Research, German Cancer Research Center (DKFZ), Heidelberg, Germany. ${ }^{32}$ Division of Preventive Oncology, German Cancer Research Center (DKFZ) and National Center for Tumor Diseases (NCT), Heidelberg, Germany. ${ }^{33}$ German Cancer Consortium (DKTK), German Cancer Research Center (DKFZ), Heidelberg, Germany. ${ }^{34}$ Department of Psychiatry and Behavioral Sciences, Stanford University, Stanford, California 94305, USA. ${ }^{35}$ Department of Biostatistics and Department of Oncology, Johns Hopkins University, Baltimore, Maryland 21205, USA.

Correspondences to: Jianxin Shi (Jianxin.Shi@nih.gov) and Nilanjan Chatterjee (nilanjan@jhu.edu) 


\begin{abstract}
Recent heritability analyses have indicated that genome-wide association studies (GWAS) have the potential to improve genetic risk prediction for complex diseases based on polygenic risk score (PRS), a simple modelling technique that can be implemented using summary-level data from the discovery samples. We herein propose modifications to improve the performance of PRS. We introduce threshold-dependent winner's-curse adjustments for marginal association coefficients that are used to weight the SNPs in PRS. Further, as a way to incorporate external functional/annotation knowledge that could identify subsets of SNPs highly enriched for associations, we propose variable thresholds for SNPs selection. We applied our methods to GWAS summary-level data of 14 complex diseases. Across all diseases, a simple winner's curse correction uniformly led to enhancement of performance of the models, whereas incorporation of functional SNPs was beneficial only for selected diseases. Compared to the standard PRS algorithm, the proposed methods in combination led to notable gain in efficiency (25-50\% increase in the prediction $\mathrm{R}^{2}$ ) for 5 of 14 diseases. As an example, for GWAS of type 2 diabetes, winner's curse correction improved prediction $\mathrm{R}^{2}$ from $2.29 \%$ based on the standard PRS to $3.10 \%(P=0.0017)$ and incorporating functional annotation data further improved $\mathrm{R}^{2}$ to $3.53 \%$ $\left(P=2 \times 10^{-5}\right)$. Our simulation studies illustrate why differential treatment of certain categories of functional SNPs, even when shown to be highly enriched for GWAS-heritability, does not lead to proportionate improvement in genetic risk-prediction because of non-uniform linkage disequilibrium structure.
\end{abstract}

Keywords: winner's curse correction, polygenic risk score, genome-wide association studies, genetic risk prediction 


\section{Introduction}

2 Large genome-wide association studies (GWAS) have accelerated the discovery of dozens or even hundreds of common single nucleotide polymorphisms (SNPs) associated with individual

4 complex traits and diseases, such as height ${ }^{1 ; 2}$, body mass index ${ }^{3}$ and common cancers (e.g.,

5 breast $^{4}$ and prostate ${ }^{5}$ cancers). Although individual SNPs typically have small effects, cumulative

6 results have provided insight about underlying biologic pathways and for some common diseases

7 like breast cancer have yielded levels of risk-stratification that could be useful as part of

8 prevention efforts ${ }^{6}$. Analyses of GWAS heritability using algorithms such as GCTA ${ }^{7 ; 8}$ have

9 shown that common SNPs have the potential to explain substantially larger fraction of the variation of many traits.

The future yield of GWAS studies, for both discovery and prediction, depends heavily on the underlying effect-size distribution (ESD) of susceptibility SNPs ${ }^{9 ; 10,6}$. A number of alternative types of analyses of ESD now point towards a polygenic architecture for most complex traits, in which thousands or even tens of thousands of common SNPs, each with small estimated effect sizes together can explain a substantial fraction of heritability ${ }^{11 ; 12}$. Mathematical analyses of power indicates that because of the polygenic nature of complex traits, future studies will need

17 large sample sizes, often by an order of magnitude higher than even some of the largest studies to 18 date, for improving accuracy of genetic risk-prediction ${ }^{10 ; 11}$. Nevertheless, for current datasets, there remains an opportunity to develop more efficient algorithms for improving the models.

21 complexity. The simplest of these methods, widely implemented in large GWAS, selects SNPs

22 based on a threshold for the significance of the marginal association test-statistics and then the

23 cumulative weighting of these SNPs by their estimated marginal strength of association is 
24 applied $^{13}$. The threshold for SNP selection can be optimized to improve the predictive

25 performance in an independent validation dataset. For a number of traits with large GWAS

26 sample sizes, it has been shown that an optimally selected threshold can improve risk prediction

27 compared to that based on the genome-wide significance threshold used for discovery ${ }^{14}$. A

28 number of newer methods involving the joint analysis of all SNPs using sophisticated mixed-

29 effect modeling techniques have recently been developed and may lead further increases in

30 model performance $\mathrm{e}^{15-17}$.

31 In this report, we propose simple modifications to the widely used PRS modeling techniques

32 using only GWAS summary-level data. Drawing from the lasso ${ }^{18}$ algorithm, we propose a simple

33 threshold dependent winner's curse adjustment for marginal association coefficients that can be

34 used to weight the SNPs in PRS. Second, to exploit external functional knowledge that might

35 identify subsets of SNPs highly enriched for association signals, we consider using multiple

36 thresholds for SNPs selection based on group membership and identify an optimal set of

37 thresholds through an independent validation dataset. We demonstrated the value of our new

38 method using summary-level results from large GWAS across a spectrum of traits, some with

39 available independent validation datasets to assess the performance of these methods. Available

40 resources, such as annotation databases, expression and methylation quantitative trait locus

41 (QTL) analyses were employed to identify groups of SNPs that are likely to be enriched with the

42 trait of interest. We evaluated the utility of this information for risk-prediction for respective

43 outcomes. We also report on the performance of new algorithm using simulation studies that

44 incorporate realistic genetic architecture, linkage disequilibrium pattern and enrichment factor

45 for underlying functional SNPs. 
48 Let $Z_{m}, P_{m}, \hat{\beta}_{m}$, and $\hat{\sigma}_{m}(m=1, \cdots, M)$ denote the univariate $Z$-statistics, the two-sided $P$ -

49 values, the estimated association coefficients and their standard deviations available as part of summary-level results for $M$ SNPs from a GWAS. We assume that each genotypic value is

51 normalized to have mean zero and unit variance and that $\hat{\beta}_{m}$ is rescaled to correspond to the

52 normalized genotypic values. Let $g_{\text {im }}$ be the genotype of SNP $m$ for subject $i$. The simplest and most popular form of the PRS for GWAS has the form

$$
P R S_{i}(\alpha)=\sum_{m=1}^{M} \hat{\beta}_{m} I\left(P_{m}<\alpha\right) g_{\text {im }}=\sum_{m=1}^{M} \hat{\beta}_{m} I\left(\mid \hat{\beta}_{m}>\lambda\right) g_{\text {im }}
$$

55 where the threshold $\alpha$ for the $P$-values, or equivalently $\lambda=\Phi^{-1}(1-\alpha / 2) \hat{\sigma}_{m}$ in the $\beta$-scale

56 (Appendix A), can be chosen to optimize the predictive performance of PRS in an independent

57 validation dataset. Here, $I(\cdot)$ is an indicator function and $\Phi(\cdot)$ is the cumulative density function

58 of the standard normal distribution.

59 Motivated from the simplification of the popular machine learning algorithm lasso ${ }^{18}$ in the

60 orthonormal case, we propose considering a lasso-type thresholding for constructing PRS in the

61 form

62

$$
P R S_{i}^{\text {lasso }}(\lambda)=\sum_{m=1}^{M} \hat{\beta}_{m}^{\text {lasso }}(\lambda) I\left(\mid \hat{\beta}_{m}>\lambda\right) g_{\text {im }}=\sum_{m=1}^{M} \operatorname{sign}\left(\hat{\beta}_{m}\right) \| \hat{\beta}_{m}|-\lambda| I\left(\left|\hat{\beta}_{m}\right|>\lambda\right) g_{\text {im }} .
$$


63 The adjustment of the association coefficient by the threshold parameter in the form of a

64 location-shift can be viewed as a "winner's curse" bias correction due to nature of the selection

65 of the SNPs. We also considered a more formal approach to winner's curse bias-correction ${ }^{19}$ by

66 maximizing a conditional likelihood $P\left(\hat{\beta}_{m}\left|\hat{\beta}_{m}\right|>\lambda\right)$ (Appendix A). Let $\hat{\beta}_{m}^{m l e}(\lambda)$ be the

67 maximum likelihood estimate of $\beta_{m}$ based on the conditional likelihood. We propose a PRS as

$$
P R S_{i}^{m l e}(\lambda)=\sum_{m=1}^{M} \hat{\beta}_{m}^{m l e}(\lambda) I\left(\left|\hat{\beta}_{m}\right|>\lambda\right) g_{i m} .
$$

It is, however, critical that for selection of the optimal threshold parameter, bias correction is performed simultaneously with SNP selection for different values of the threshold parameters.

71 We have previously studied the theoretical power for use of such lasso-type winner's curse correction for developing PRS when SNPs are independent and concluded that under realistic polygenic architecture this simple correction has the potential to improve predictive performance of PRS ${ }^{11}$. The performance of such an algorithm in real GWAS data, where independent SNPs need to be selected after linkage disequilibrium (LD)-filtering, has not been evaluated.

Information from various functional studies, annotation databases and GWAS from various traits

77 is increasingly available to allow identification of subset of SNPs that can be considered to have

78 potential high-prior probability for association with a given trait. Various types of enrichment

79 analyses, whether based on distribution of summary-level statistics ${ }^{20}$ or on more advanced

80 heritability-partitioning analyses ${ }^{21 ; 22}$, have shown empirical evidence of strong enrichment of

81 GWAS association signals for different categories of SNPs which represent only a relatively

82 small fractions of all GWAS SNPs. However, very few systematic studies have examined

83 whether and how such enrichment information can be utilized to improve models for genetic risk 
84 prediction. We consider a simple modification to PRS to explore this issue. We assume that the

85 set of $M$ SNPs can be partitioned into two mutually exclusive groups, $S_{1}$ and $S_{2}$, where $S_{1}$

86 represents a relatively small subset representing "high-prior" SNPs (referred to as HP) and the

87 second group $S_{2}$ represents the remainder of the GWAS SNPs (referred to as "low-prior" SNPs

88 or LP) that can be considered part of an "agnostic" search. We allow differential treatment of the

89 SNPs in the PRS:

90

$$
P R S_{i}\left(\lambda_{1}, \lambda_{2}\right)=\sum_{m \in S_{1}} \tilde{\beta}_{m} I\left(\left|\hat{\beta}_{m}\right|>\lambda_{1}\right) g_{i m}+\sum_{m \in S_{2}} \tilde{\beta}_{m} I\left(\left|\hat{\beta}_{m}\right|>\lambda_{2}\right) g_{i m}
$$

and select the optimal set of threshold parameters based on independent validation dataset(s). We

92 refer to the PRS selecting SNPs with two separate thresholds as two-dimensional PRS or 2D

93 PRS. Here, $\widetilde{\beta}_{m}$ can be chosen as the original estimate $\hat{\beta}_{m}$, the lasso-type correction

$94 \hat{\beta}_{m}^{\text {lasso }}=\operatorname{sign}\left(\hat{\beta}_{m}\right)\left(\left|\hat{\beta}_{m}\right|-\lambda\right)^{+}$or the MLE correction, $\hat{\beta}_{m}^{m l e}$. The PRS in (1), (2) and (3) using a

95 single threshold is referred to as 1D PRS.

96 Following analytic techniques similar to those derived for $1 \mathrm{D} \mathrm{PRS}^{11}$, we can characterize the

97 theoretical predictive performance of 2D PRS and the corresponding optimal set of thresholds

98 based on the genetic architecture parameters of the two sets of SNPs assuming independence

99 (Appendix B). Using simulation studies, we study performance of the method with realistic LD

100 pattern among SNPs.

\section{$101 \quad$ LD-pruning and LD-clumping}

102 The performance of PRS is typically improved if genetic markers are pruned for $\mathrm{LD}^{23}$. LD-

103 pruning procedures that ignore GWAS $P$-values frequently prune out the most significant SNPs 
104 and may reduce performance. Instead, we use the LD-clumping procedure implemented in

105 PLINK ${ }^{23}$ that chooses the most significant SNP from a set of SNPs in LD guided by GWAS $P$ -

106 values. After LD-clumping, no SNPs with physical distance less than 500kb have LD $r^{2} \geq 0.1$.

107 Expanding HP SNP set through LD

108 Suppose $S_{I}$ is a given HP set defined based on external annotation data (see section Annotation

109 datasets). Any SNP in high LD with a SNP in $S_{l}$ is also considered to be an HP SNP. Thus, we

110 expanded $S_{l}$ by including all SNPs that were in high LD $\left(r^{2} \geq 0.8\right)$ with any SNP in the original

$111 S_{1}$

112 Simulation Scheme

113 We performed simulations to evaluate the performance of six PRS prediction methods: 1D and

114 2D PRS without winner's curse correction and with lasso/MLE winner's curse correction. To

115 make simulations realistic in terms of the distribution of minor allele frequencies (MAF) and LD,

116 we simulated quantitative traits with specific genetic architecture by conditioning on the

117 genotypes of a lung cancer $\mathrm{GWAS}^{24}$, which had 11,924 samples of European ancestry and

118485,315 autosomal SNPs after quality control. The simulation scheme is summarized in the

119 following steps:

120 (1) We performed LD-pruning implemented in PLINK so that no SNPs within 500kb were in

121 LD at threshold $r^{2}=0.1$. After LD-pruning, $M=53,163$ autosomal SNPs (denoted as

$122 S)$ were left.

123 (2) Denote $S_{1}$ as the putative HP SNP set and $S_{2}=S \backslash S_{1}$ as the LP SNP set. We selected a 124 set of 5000 "causal" SNPs (denoted as $C$ ) from the pruned SNP set $S$. If $C$ is randomly 
selected, i.e., $S_{1}$ is not enriched with causal SNPs, we expect $\left|S_{1} \cap C\right|=\left|C \| S_{1}\right| / M \mathrm{SNPs}$

$$
\Delta=\frac{\left|S_{1} \cap C\right|}{\left|C \| S_{1}\right| / M}
$$

(3) We simulated quantitative traits according to $y_{i}=\sum_{t \in C} \beta_{t} g_{i t}+\varepsilon_{i}$, where $\beta_{t} s$ were simulated independently from a Gaussian mixture distribution $\beta_{t} \sim \pi N\left(0, \sigma_{1}^{2}\right)+(1-\pi) N\left(0, \sigma_{2}^{2}\right)$ with $\pi=0.1$. Here, $\sigma_{1}^{2}, \sigma_{2}^{2}$ and $\operatorname{Var}\left(\varepsilon_{i}\right)$ were scaled so that $\operatorname{Var}\left(y_{i}\right)=1$. The phenotypic variances explained by the two components were $h_{1}^{2}=|C| \pi \sigma_{1}^{2}=0.1$ and $h_{2}^{2}=|C|(1-\pi) \sigma_{2}^{2}=0.4$. We assume the same effect-size distribution for both HP and LP causal SNPs, but the proportions of causal SNPs are higher in the former than the later group. Under this assumption, $\Delta$ also reflects the ratio of heritability explained at a per SNP basis in the HP set compared to LP set.

(4) We randomly selected 10,000 samples as a discovery set and 1,924 as a validation set. We performed GWAS association analysis for all 485,315 autosomal SNPs in the discovery sample. The summary statistics were used to calculate PRS for each sample in the validation sample. The prediction $R^{2}$ was calculated as $\max _{\lambda} \operatorname{cor}^{2}\left(P R S_{i}(\lambda), y_{i}\right)$ for 1D PRS methods and $\max _{\lambda_{1}, \lambda_{2}} \operatorname{cor}^{2}\left(P R S_{i}\left(\lambda_{1}, \lambda_{2}\right), y_{i}\right)$ for 2D PRS methods. We repeated the simulation 50 times for each set of parameters and report the average prediction $R^{2}$. 
143 Recently, Finucane et al. ${ }^{22}$ reported the heritability explained by common SNPs in multiple

144 functional categories for 17 traits. Interestingly, they found that common SNPs located in regions

145 that are conserved in mammals ${ }^{25}$ accounted for about $2.6 \%$ of total common SNPs but explained

146 approximately $35 \%$ of total heritability in average across these traits, suggesting a 13.5 -fold

147 enrichment. Thus, we were motivated to investigate whether SNPs related with the conserved

148 regions (CR) may be useful for 2D PRS methods. We downloaded the CR annotations (see Web

149 Resources), identified common SNPs located in any CR and also identified their LD SNPs with

$150 r^{2} \geq 0.8$. These SNPs are referred as CR-SNPs, which were used as HP $S_{1}$ in simulations. We

151 found 9,940 CR-SNPs overlapping with the 53,163 LD-pruned SNPs. To investigate whether

152 specific genomic locations of CR-SNPs influence the performance of 2D-PRS, we also

153 performed simulations using a set $S_{1}$ of random SNPs that has the same size and associated

154 heritability as the CR-SNPs. 
157 The information for GWAS data sets and functional annotation data are summarized in Tables $158 \quad$ S1A and S1B.

\section{WTCCC GWAS data}

The Wellcome Trust Case Control Consortium ${ }^{26}$ (WTCCC) data consisted of two control data sets (1958 Cohort samples and NBS control samples) and seven diseases: bipolar disorder (BD), coronary artery disease (CAD), Crohn's disease (CD), hypertension (HT), rheumatoid arthritis (RA), Type 1 diabetes (T1D) and Type 2 diabetes (T2D). Since we analyzed T2D using a much larger discovery sample, we did not analyze the T2D data in WTCCC. Because cases and controls were genotyped in different batches, differential errors between cases and controls might cause a serious overestimate of the risk prediction. Thus, we performed very rigorous

167 quality control (QC) by removing duplicate samples, first or second degree relatives, samples

168 with missing rate greater than $5 \%$ and non-European samples identified from EigenStrat ${ }^{27}$ 169 analysis. For each disease, we excluded SNPs with $\mathrm{MAF}<5 \%$, missing rate $>2 \%$, missing rate 170 difference $>1 \%$ between cases and controls or $P_{\mathrm{HWE}}<10^{-4}$ in the control samples. After QC, we

171 had 2,928 controls, 1,817 BD cases, 1,878 CAD cases, 1,729 CD cases, 1,934 HT cases, 1,894

172 RA cases and 1,939 T1D cases. For each PRS method and each disease, we estimated the 173 prediction $\mathrm{R}^{2}$ by five-fold cross-validation.

\section{Three cancer GWAS with individual genotype data}

175 We analyzed three cancer GWAS with individual level genotype data: the bladder cancer ${ }^{28 ; 29}$

176 GWAS of European ancestry including 5,937 cases and 10,862 controls, the pancreatic cancer

$177 \mathrm{GWAS}^{30}$ of European ancestry (after excluding samples with Asian or African ancestry) 
178 including 5,066 cases and 8,807 controls, and the Asian non-smoking female lung cancer

179 GWAS $^{31}$ with 5,510 cases and 4,544 controls. After QC, the bladder cancer GWAS had 463,559 autosomal SNPs and the Asian lung cancer GWAS had 329,703 autosomal SNPs. The pancreatic cancer GWAS included samples from three studies that used different genotyping platforms. For convenience, we analyzed 267,935 autosomal SNPs that overlapped in all three platforms. The prediction performance was evaluated using ten-fold cross-validation.

Five large GWAS with summary statistics and independent validation samples

For T2D, we downloaded the summary statistics of the DIAGRAM (DIAbetes Genetics

Replication And Meta-analysis) consortium ${ }^{32}$ with 12,171 cases and 56,862 controls for 2.5

7,131 T2D patients and 49,747 samples without T2D (but may have other medical conditions, depression). Although these non-T2D samples were not perfect healthy controls, we found that most of the genome-wide significant SNPs in DIAGRAM could be replicated in GERA (data not 193 shown). We randomly selected 5,631 T2D patients and 48,247 non-T2D subjects from GERA as discovery set, performed association analysis adjusting for top 10 PCA scores and meta-analyzed with the summary statistics from DIAGRAM for 353,196 autosomal SNPs overlapping between the two studies. The resulting summary statistics were used to build PRS risk models, which were validated in the remaining 1500 T2D patients and 1500 non-T2D subjects in GERA.

198 The PGC2 (Psychiatric Genetics Consortium) schizophrenia GWAS meta-analysis consisted of 19934,241 cases and 45,604 controls ${ }^{33}$. Summary statistics were obtained by meta-analyzing all 
200

201

202

203

204

205

206

207

208

209

210

211

212

213

214

215

216

217

218

219

220

221

PGC2 schizophrenia GWAS except the MGS $^{34}$ (Molecular Genetics of Schizophrenia) subjects of European ancestry. The summary statistics were used to build PRS models, which were validated in MGS samples with 2,681 cases and 2653 controls.

The TRICL (Transdisciplinary Research in Cancer of the Lung) GWAS consortium consisted of 12,537 lung cancer cases and 17,285 controls $^{35 ; 36}$. We performed meta-analysis using TRICL samples excluding the samples from the $\mathrm{PLCO}^{24}$ (Prostate, Lung, Colon, and Ovary Cohort Study) study. The summary statistics based on 11,300 cases and 15,952 controls were used to build risk models, which were validated in the PLCO lung GWAS samples with 1,237 cases and 1,333 controls.

For colorectal cancer, we performed meta-analysis for the GECCO (Genetics and Epidemiology of Colorectal Cancer Consortium) ${ }^{37}$ GWAS data after excluding the PLCO GWAS data. The PLCO samples were genotyped using two different genotyping platforms with different marker densities: one had approximately 500K SNPs and the other had only 250K SNPs. Thus, we first imputed the genotypes to the Hapmap2 reference panel using IMPUTE2 ${ }^{38}$ and selected SNPs with imputation $r^{2} \geq 0.9$ for risk prediction. The discovery sample consisted of 9,719 cases and 10,937 controls from 19 studies. The PLCO validation sample had 1,000 cases and 2,302 controls.

The summary statistics for prostate cancer were obtained from the PRACTICAL (PRostate cancer AssoCiation group To Investigate Cancer Associated aLterations) consortium and The GAME-ON/ELLIPSE (Elucidating Loci Involved in Prostate Cancer Susceptibility) Consortium with samples from populations of European, African, Japanese and Latino ancestry ${ }^{5}$. The discovery samples consisted of 38,703 cases and 40,796 controls after excluding the NCI Pegsus 
222 GWAS samples with 4,600 cases and 2,941 controls, which were used for validation. We

223 analyzed 536,057 autosomal SNPs after QC that overlapped between the validation and the

224 discovery sample summary statistics.

225

226

227

228

229

230

231

232

233

234

235

236

237

238

239

240

241

242

243

\section{$\underline{\text { Annotation datasets }}$}

For many traits, GWAS risk SNPs have been reported to show enrichment for eQTLs, methylation QTLs (meQTLs) and cis-regulatory elements (CREs). In addition, recent studies have reported extensive genetic pleiotropy across diseases and traits, e.g. psychiatric diseases ${ }^{39 ;}$ 40, schizophrenia and cardiovascular-disease risk factors, including blood pressure, triglycerides, low- and high-density lipoprotein, body mass index (BMI) and waist-to-hip ratio (WHR) ${ }^{41}$. Thus, we defined the HP SNP set $S_{1}$ using eQTL SNPs (referred to as eSNPs) in blood, tissue specific eSNPs and meQTL SNPs (referred to as meSNPs), SNPs related with CREs (referred to as CRESNPs), SNPs related with genomic regions conserved across mammals (referred to as CR-SNPs) and SNPs identified by pleiotropic analyses (referred to as PT-SNPs). We expanded each SNP set by including LD SNPs with $r^{2} \geq 0.8$ in the local $1 \mathrm{M}$ region for each SNP. Here, LD was calculated based on the genotype data of relevant ancestry in The 1000 Genomes Project ${ }^{42}$.

eSNPs and meSNPs: Blood cis-eSNPs were identified from two large-scale eQTL studies in European populations. One study involved a transcriptome sequencing project of 922 subjects ${ }^{43}$ and the other involved a microarray study of 5,311 subjects ${ }^{44}$. Because of its very large sample size, the second study had the power to detect eSNPs with even tiny effect sizes which may not have meaningful functional importance. Thus, we included eSNPs with association $P$-value $<10^{-6}$ with any gene in the cis region in the second study. For both Asian and European lung cancer GWAS data, we used eSNPs ${ }^{45}$ and meSNPs ${ }^{46}$ based on lung tissues. For T2D, we used eSNPs ${ }^{47}$ 
244 and meSNPs ${ }^{48}$ based on adipose tissues. Furthermore, detected trans-SNPs are much fewer than

245 cis-SNPs and the replication rate of trans-eSNPs was much lower than cis-SNPs $^{47}$, suggesting

246 that including trans-SNPs would be unlikely to improve risk prediction. Thus, we did not include

247 trans-SNPs.

248 CRE-SNPS: CREs are regions of noncoding DNA regulating the transcription of nearby genes.

249 SNPs located in CREs may change the binding of specific transcription factors and thus the

250 expression of the target genes. Typically, CREs are identified through ChIP-Seq experiments of

251 histone modifications. We downloaded "peak" data (each peak represents one CRE) of specific

252 sets of histone methylation markings, acetylation markings and DNase I hypersensitive sites

253 (DHSs) from the ROADMAP project website for relevant cell lines. For each identified CRE

254 ('peak'), we identified common SNPs with MAF>1\%. For prostate cancer, we used the ChIP-

255 Seq data for H3K27Ac and the transcription factor TCF7L2 ${ }^{49}$ to define HP SNP sets.

$256 \quad$ PT-SNPS: The summary statistics for height ${ }^{1 ; 2}, \mathrm{BMI}$ and obesity ${ }^{3 ; 50}, \mathrm{WHR}^{51}$, waist

257 circumference $(\mathrm{WC})^{51}$, hip circumference (HIP) ${ }^{51}$ were downloaded from the GIANT consortium

258 website. The summary statistics for GWAS meta-analysis of cardiovascular-disease risk

259 factors $^{52}$, including triglycerides (TG), low-density lipoprotein (LDL) and high-density

260 lipoprotein (HDL), were also used for 2D PRS.

261 We investigated whether or not each tentative HP SNP set was enriched for GWAS associations

262 by examining the quantile-quantile (QQ) plot, which was made for HP SNPs vs. LP SNPs after

263 LD-clumping. The SNP sets not enriched for GWAS associations were not expected to improve

264 risk prediction in 2D PRS. Thus, for each disease, we only included HP SNP sets for 2D PRS

265 when they showed strong enrichment in QQ plots. Interestingly, blood eSNPs were enriched for 
266

267

268

269

270

271

272

273

274

275

276

277

278

279

280

281

almost all diseases. CR-SNPs showed modest enrichment for majority of the diseases. Thus, blood eSNPs and CR-SNPs were used for 2D PRS for all diseases. In addition, eSNPs and meSNPs derived in lung tissues were enriched in lung cancer GWAS of both European and Asian ancestry. The SNPs related in enhancer and active promoter regions (characterized by H3K4me3, H3K9-14Ac, H3K36me3, H3K4me1, H3K9ac and H3K9me3) were enriched for GWAS associations but SNPs related with the repressive regions (characterized by H3K27me3) were not. Thus, we included SNPs related with these enhancer and active promoter regions for 2D PRS. DHS SNPs were not strongly enriched and thus were excluded. Recently, we have shown significantly shared genetic component between lung cancer and bladder cancer risk ${ }^{53}$. Thus, we also used HP SNPs derived based on lung tissues or cell lines for predicting bladder cancer risk. Furthermore, we found that SNPs identified through pleiotropic analysis were enriched in multiple diseases. For example, SNPs with $P$-value $<0.001$ in GWAS of height, HDL, LDL, TC, TG, WC, obesity, HIP and T2D were enriched in lung cancer GWAS. Because our 2D PRS methods required a relatively large number of HP SNPs to achieve improvement, we combined the SNPs with $P$-value $<10^{-3}$ (or $10^{-2}$ ) in at least one trait into a HP SNP set referred as PT-0.001 (or PT-0.01).

\section{Testing the statistical significance of improvement for risk prediction}

For WTCCC and three cancer GWAS data sets with individual genotype data, we used K-fold cross-validation to estimate prediction $\mathrm{R}^{2}$. Here, $K=5$ for WTCCC data and $K=10$ for cancer GWAS data. We were interested in testing whether the prediction of a new PRS method was significantly better than that of the standard 1D PRS defined in equation (1). For the $i^{\text {th }}$ crossvalidation, we denote $R_{i, 0}^{2}$ as the maximum prediction for the standard 1D PRS optimized across 
288

289

290

291

292

293

$P$-value thresholds, $R_{i, 1}^{2}$ as the maximum prediction for a new PRS method optimized across all $P$-value thresholds for 1D PRS and all pairs of $P$-value thresholds for 2D PRS. We defined $\delta_{i}=R_{i, 1}^{2}-R_{i, 0}^{2}$ and estimated its variance as $\hat{\sigma}^{2}=\sum_{i=1}^{K}\left(\delta_{i}-\bar{\delta}\right)^{2} /(K-1)$ with $\bar{\delta}=\sum_{i=1}^{K} \delta_{i} / K$.

We calculated the statistic $T=\bar{\delta} / \sqrt{\hat{\sigma}^{2} / K}$ and evaluated its significance using the $t$-distribution. For the five diseases with independent validation samples, we used bootstrap to estimate the variance of the $\mathrm{R}^{2}$ estimates to test significance ${ }^{42}$.

\section{Results}

\section{Theoretic investigation of 2D PRS performance assuming independent SNPs}

Figure 1A shows the theoretically-derived AUC for a binary trait based on 1D PRS and 2D PRS without applying a winner's curse correction. For all PRS models, the AUC increases with the sample size of the discovery dataset. The 2D PRS can improve the 1D PRS in which the magnitude depends on the sample size in the discovery sample and also the enrichment of the HP SNPs. Figure 1B shows the optimal $P$-value thresholds for including SNPs that maximize the prediction of 2D PRS. The optimal P-value threshold for including HP SNPs is more liberal than that for LP SNPs and the difference diminishes as the training sample size becomes very large.

\section{Polygenic risk prediction of T2D}

Figure 2A presents the 1D PRS results for T2D. The standard 1D PRS without winner's curse correction had a prediction $\mathrm{R}^{2}=2.29 \%$ by including SNPs with $\mathrm{P} \leq 2 \times 10^{-3}$. The winner's curse correction improved $\mathrm{R}^{2}$ to $3.10 \%$ using the lasso-type correction and 2.67\% using the MLE correction. 
Next, we investigated whether functional annotation could further improve risk prediction. We considered CR-SNPs, eSNPs and meSNPs in adipose tissue, and SNPs related with different histone marks and their combinations as HP SNP sets. These SNPs were enriched in T2D GWAS, exemplified by the QQ plot in Figure 2B for a HP SNP set comprising of eSNPs/meSNPs in adipose tissue and SNPs related with $\mathrm{H} 3 \mathrm{~K} 4 \mathrm{me} 3$ in the pancreatic islet cell line. Note that the SNPs have been pruned to have LD $r^{2} \leq 0.1$, so the observed enrichment was unlikely due to an artifact related to extensive LD. Figure $2 \mathrm{C}$ illustrates how the prediction $\mathrm{R}^{2}$ of a 2D PRS depends on the P-value thresholds for the HP and LP SNPs. The prediction $\mathrm{R}^{2}$ was maximized using a more liberal $P$-value threshold 0.03 for HP SNPs and a more rigorous threshold 0.005 for LP SNPs. This optimal 2D PRS had 8,018 HP SNPs and 2,033 LP SNPs.

318 Figure 2D reports the prediction $\mathrm{R}^{2}$, AUC and the significance for testing of whether an 319 alternative PRS method could improve the standard 1D PRS. The best predictions were achieved 320 by the $2 \mathrm{D}$ PRS with lasso-type correction: $\mathrm{R}^{2}=3.48 \%$ using eSNPs/meSNPs and CR-SNPs and $\mathrm{R}^{2}=3.53 \%$ using eSNPs/meSNPs and H3K4me3 SNPs in pancreatic islet cell line $(52.0 \%$ and

$32254.1 \%$ efficiency gain compared to $2.29 \%$ using standard 1D PRS, respectively). These

323 improvements were statistically significant compared to the $1 \mathrm{D}$ standard PRS $(P=0.00002$ and

324 0.00004, respectively). Of note, the recently developed method LD-pred ${ }^{54}$ that models the LD

325 information only slightly improved prediction $\mathrm{R}^{2}$ from $2.47 \%$ to $2.73 \%$ (10.5\% efficiency gain)

326 using DIAGRAM summary statistics as discovery. 


\section{Results for WTCCC data}

The prediction $\mathrm{R}^{2}$ values for six diseases in WTCCC data are reported in Figure 3A. The AUCs and Nagelkerke $\mathrm{R}^{2}$ are summarized in Table S2. Optimal thresholds for SNP selection are in Table S3. The lasso-type winner's curse correction improved the 1D PRS predictions for CD (6.65\% to $8.22 \%)$, RA (7.24\% to $8.60 \%)$ and T1D (18.2\% to $18.5 \%)$ and was slightly better than the MLE winner's curse correction. The 2D PRS improved the prediction for CD (6.65\% to method of winner's curse correction together 2D PRS performed at least as well as the standard 1D PRS. However, because of the small sample size in the validation sample, the improvements were not statistically significant.

\section{Results for three cancer GWAS with individual genotype data}

Results are summarized in Figure 3B (prediction $\mathrm{R}^{2}$ ), Table S4 (AUC and Nagelkerke $\mathrm{R}^{2}$ ), Table S5 ( $P$-value for testing significance of improvement) and Table S6 (optimal thresholds for SNP selection). The standard 1D PRS achieved an $\mathrm{R}^{2}=1.12 \%$ for bladder cancer, $2.35 \%$ for Asian nonsmoking female lung cancer and $2.2 \%$ for pancreatic cancer, indicating the difficulty of genetic risk prediction for these cancers. The lasso-type correction improved the 1D PRS for all

345 three cancers: $\mathrm{R}^{2}$ from $1.12 \%$ to $1.29 \%$ for bladder cancer, $2.35 \%$ to $2.51 \%$ for Asian female nonsmoking lung cancer and $2.20 \%$ to $2.54 \%$ for pancreatic cancer. Our 2D PRS methods

347 further improved the prediction although the various annotation datasets gave different

348 improvement. For bladder cancer, the greatest efficiency gain $\left(\mathrm{R}^{2}=1.64 \%, 46.4 \%\right.$ efficiency gain 
350

351

352

353

354

355

356

357

358

359

360

361

362

363

364

365

366

367

368

369

370

371

correction) was achieved with the SNPs related to the lung tissue/cell line expression data (eSNPs, meSNPs, H3K4me3 SNPs in SAEC), which performed slightly better than the SNPs related with histone marks in bladder cell line $\left(\mathrm{R}^{2}=1.46 \%\right)$. For non-smoking female Asian lung cancer, the 2D PRS incorporated with PT-0.001 SNPs or H3K4me3 SNPs in HAEC improved $\mathrm{R}^{2}$ to $2.84 \%$. For pancreatic cancer, the 2D PRS incorporated with CR-SNPs, SNPs related with histone marks of pancreatic islet and adipose eSNPs/meSNPs improved prediction $\mathrm{R}^{2}$ by approximately $\sim 30 \%$ compared with the standard 1D PRS. Many of the improvements over the standard 1D PRS were statistically significant (Table S5), e.g., $\mathrm{P}=0.025$ for 2D PRS with H3K4me3 SNPs in HAEC for bladder cancer, P=0.025 for 2D PRS with PT-0.001 SNPs for Asian lung cancer and $\mathrm{P}=0.047(0.023,0.023)$ for $2 \mathrm{D}$ PRS with CR-SNPs (PT-0.001, PT-0.01 SNPs) for pancreatic cancer.

\section{Results for four large-scale summary-statistics datasets}

Prediction results are reported in Figure 3C (prediction $\mathrm{R}^{2}$ ), Table S7 (AUC and Nagelkerke $\mathrm{R}^{2}$ ), Table S8 ( $P$-values for testing whether improvements were significant), Table S9 (optimal pvalue thresholds for SNP selection in 2D PRS) and Figure S2. For lung cancer, the standard 1D PRS had an $\mathrm{R}^{2}=1.13 \%$. The best prediction $\mathrm{R}^{2}=1.65 \%$ (a $46.0 \%$ efficiency gain compared with the standard 1D PRS) was achieved by lasso-corrected 2D PRS with eSNPs/meSNPs in lung tissues, blood eSNPs and SNPs related with H3K4me3 in SAEC. To achieve this prediction accuracy, the optimal $P$-value threshold for the 2 D PRS should be 0.008 for HP SNPs and $5 \times 10^{-6}$ for LP SNPs. However, the improvement was not statistically significant. For schizophrenia, the lasso-type correction improved 1D PRS R ${ }^{2}$ from $14.01 \%$ to $14.94 \%$; the 2D PRS with CR-SNPs further improved the $\mathrm{R}^{2}$ to $15.37 \%$ and the improvement was highly statistically significant 
$372\left(\mathrm{P}=3.2 \times 10^{-10}\right)$. The optimal $\mathrm{p}$-value threshold was 0.6 for CR-SNPs and 0.1 for other SNPs in 2D

373 PRS with lasso-type correction. For CRC and prostate cancer, neither winner's curse correction

374 nor 2D PRS improved prediction.

375

Simulation results

377 The simulation results are summarized in Figure 4. First, the winner's curse corrections, both lasso-type correction and MLE correction, slightly improved prediction in most if not all simulations and in particular improved more for the 1D PRS than the 2D PRS. We also observed that the two winner's curse correction methods performed similarly. Second, if HP SNPs were chosen randomly in the LD-pruned SNP set and were strongly enriched for causal SNPs, the 2D PRS methods substantially improved the prediction over the 1D PRS methods. As expected, the improvement increased quickly with the enrichment fold change $\Delta$. Without winner's curse correction, 1D PRS had $\mathrm{R}^{2}=1.38 \%$ and $2 \mathrm{D}$ PRS improved $\mathrm{R}^{2}$ to $2.13 \%$ for $\Delta=2,2.86 \%$ for $\Delta=3$ and $4.22 \%$ for $\Delta=4$. Consistent with theoretical analysis assuming independent SNPs (Figure 1B), the optimal $P$-value threshold for HP SNPs was more liberal than that for LP SNPs (Table S10). compared to the simulations with randomly selected HP SNPs, even with the same enrichment

390 fold change. As a numerical example, when $\Delta=4$, the 2D PRS method without winner's curse 391 correction improved $\mathrm{R}^{2}$ from $1.38 \%$ to $1.73 \%$ for CR-SNPs as HP SNPs while from $1.38 \%$ to 4.22\% for random HP SNPs. To investigate whether the difference was caused by different local LD structure, for each SNP, we counted the number of SNPs located less than $1 \mathrm{Mb}$ from the 394 given SNP and had $r^{2} \geq 0.8$ with the SNP in the 1000 Genomes Project. For 9,940 CR-SNPs 
used for our simulations, the average number of LD SNPs is 22.4 (median=12) while the average

396 number is 6.4 (median=2) for non-CR SNPs. See also the histograms in Figure S1. Thus, CR-

397 SNPs are enriched in regions with strong LD and may suggest a possible explanation why CR-

398 SNPs (and other functional categories with similar LD structure) may not lead to improvement in risk prediction as much as would be expected based on enriched heritability.

\section{Discussion}

401 Our study demonstrates that the predictive performance of GWAS PRS models can be improved 402 based on a combination of a simple adjustment to the threshold levels of SNP selection and weights of selected SNPs. The degree of gain, however, is not uniform and depends on multiple

404 factors, including the genetic architecture of the trait, sample size of the discovery sample set, 405 degree of enrichment of association in selected set of "high-prior" SNPs and the linkage 406 disequilibrium patterns of these SNPs with the rest of the genome.

407 The simple winner's curse correction of SNP weights using the lasso-type method leads to an 408 improvement in performance of PRS uniformly across all studied diseases. For some diseases, 409 such as type-2 diabetes (Figure 2 and Table S7) or Crohn's disease (Figure 3 and Table S2), this 410 correction alone led to notable improvement in the performance of PRS. The optimal weighting

411 of SNPs would depend on the true effect size distribution of the underlying susceptibility SNPs.

412 Lasso-type weights can be expected to be optimal under a double exponential distribution ${ }^{18 ; 55}$,

413 and it is possible that the weighting could be improved further under alternative models of effect-

414 size distribution. It is, however, encouraging that irrespective of what might be the true effect415 size distribution, which is likely to vary across the diseases of study, our simple lasso-correction 
416 improves over the standard PRS methodology without adding any additional computational

417 complexity.

418 The effect of using various threshold levels for different functional categories of SNPs on the

419 performance of the model varied by disease as well as the functional annotation of external data

420 sets employed in our analytical approach. After adjustment with lasso-type weights, the use of

421 two-dimensional threshold based on prioritized SNPs led to notably higher values of $\mathrm{R}^{2}$ for lung

422 cancer in Caucasians (increase by $46 \%$ using eSNPs, meSNPs and SNPs related with H3K4me3

423 in SAEC as high priority set), bladder cancer (increased by $27.1 \%$ using high priority SNPs in

424 lung tissue or cell lines), type-2 diabetes (increased by 13.9\% using eSNPs, meSNPs and SNPs

425 related with $\mathrm{H} 3 \mathrm{~K} 4 \mathrm{me} 3$ mark in islet cell line) and pancreatic cancer (increased by $10.6 \%$ using

426 SNPs related with histone modification marks in pancreatic or islet cell lines). Consistent with

427 theoretical expectations, for each of the traits, the optimal thresholds selected were more liberal

428 for the associated category of high-prior SNPs than those for complementary set.

429 Our simulation study illustrated how the improvement in performance of the PRS model due to

430 differential treatment of certain categories of SNPs is modest even when these SNPs have been

431 categorized to be highly enriched for heritability ${ }^{21}$. For example, recent heritability partitioning

432 analysis has identified SNPs in conserved DNA regions, representing $2.6 \%$ of the genome, to be

433 highly enriched for GWAS heritability for many diseases (explaining 35\% heritability on

434 average). Our theoretical calculations suggest that if only independent SNPs are analyzed, use of

435 a subset of SNPs similarly enriched for heritability is expected to yield much higher

436 improvement in the performance of the model (Figure 1). Our simulation studies showed that a

437 similarly large gain is expected even in the presence of naturally occurring LD pattern if these 
438 SNPs are selected randomly from the genome. However, when we simulated high-prior SNPs

439 based on the exact location of the CR-SNPs, the improvement was modest, within the range of

440 observed data. The CR-SNPs represent a highly unusual linkage disequilibrium pattern in that

441 they are in high degree of LD with an unusually large number of neighboring SNPs (Figure S1).

442 In the future, more detailed and accurate assessment of the functional annotation of SNPs should

443 improve performance of PRS models. Our method requires only simple modifications to the

444 standard PRS algorithm and can thereby be used to rapidly evaluate the effectiveness of many

445 alternative strategies. In the current study, we used physical location information pertaining to

446 histone marks to define high-priority SNP. However, a SNP located in histone marks does not

447 necessarily cause the variation in histone binding. Thus, a more reasonable approach is to

448 identify genetic variants associated with histone variation across subjects in order to define high-

449 priority SNP sets. These types of histone QTLs have recently been reported in small-scale

450 studies based on HapMap samples ${ }^{56 ; 57}$. We expect that histone QTL SNPs identified in future

451 large-scale tissue specific studies might be more informative for risk prediction.

452 We have investigated the performance of the various algorithms using criteria that reflect how

453 much of the variability of the observed outcomes can be explained by the PRS in the validation

454 dataset. For clinical applications of risk-models, however, it is important to evaluate whether

455 models are well calibrated that is to what extent they can produce unbiased estimates of risk for

456 individuals with different SNP profiles. Earlier studies have noted that the standard PRS can be

457 mis-calibrated and additional calibration steps may be needed when applying PRS in a clinical

458 setting. In this regard, we find that a winner's curse correction can alleviate calibration bias of

459 the standard PRS, but substantial residual bias remains in some situations (Table S11). The 
460

461

462

463

464

465

466

467

468

469

470

471

472

473

474

475

476

477

478

479

480

regression relationship between overall PRS and disease status can be estimated based on a relatively small validation sample and can also be used to re-scale PRS for producing calibrated risk estimates.

We used several different metrics for evaluating the potential impact of an improved PRS for risk-stratification. The percentage gain in prediction $\mathrm{R}^{2}$ due to improved PRS is substantial for several diseases. For these diseases, the impact of an improved PRS on overall discriminatory performance of the models is noticeable but small (increase in AUC value between 1-2\%).

However, even a modest increment in AUC value can lead to identification of substantially higher fraction of individuals who are at the tails of risk distribution and hence likely to consider clinical decisions (Table S12).

A limitation of our method is that we use stringent LD-pruning for creating sets of independent SNPs. However, this may result in loss of predictive power of models as SNPs in moderate or low LD may still harbor independent association signals. The LD-pred ${ }^{54}$ method has been proposed to better account for correlated SNPs in building PRS using GWAS summary-level data and has been shown to lead to improved performance over standard PRS for some diseases such as schizophrenia. The LD-pred method also uses a specific form of prior distribution for obtaining "shrunken" estimates of the regression coefficients for the SNPs in the model. Although we did not make direct comparisons, it appears that the LD-pred method gains over standard PRS by improving the accounting for correlation between risk SNPs. In contrast, in our algorithm, which used stringent LD pruning, the gain in performance over the standard PRS mainly came from the lasso-type winner's curse correction and the use of variable thresholds to 
481 account for HP and LP SNPs. Thus it is possible that in the future the complementary strengths

482 of the algorithms can be combined to develop more powerful PRS.

483 In conclusion, we have proposed a set of simple methods for constructing PRS for genetic risk

484 prediction using GWAS summary-level data. The proposed methods are computationally not

485 onerous and yet show a noteworthy gain in performance. A major strength of our study is that we

486 evaluated the proposed methods across a large number of scenarios reflecting a spectrum of

487 underlying genetic architectures for different complex diseases, sample size of the study and

488 available functional annotation. These studies and additional simulations provide comprehensive

489 insights to promises and limitations of genetic risk prediction models in the near future. 


\section{Appendix A: Winner's curse correction}

\section{Lasso-type winner's curse bias correction estimator}

495 Suppose that for a given SNP, we have the two-sided P-value $P_{i}$, the regression coefficient $\hat{\beta}_{i}$, its 496 standard deviation $\hat{\sigma}_{i}$ and the $Z$-statistic $Z_{i}=\hat{\beta}_{i} / \hat{\sigma}_{i}$. The SNP is included in a risk prediction 497 model if $P_{i} \leq \alpha$ or equivalently $\left|Z_{i}\right| \geq \Phi^{-1}(1-\alpha / 2)$ or equivalently $\left|\hat{\beta}_{i}\right| \geq \lambda\left(\alpha, \hat{\sigma}_{i}\right)=$ $498 \Phi-11-\alpha / 2 \sigma i$. Here, $\Phi()$ is the cumulative distribution function of $N(0,1)$. The lasso-type 499 shrinkage estimator conditioning on $P_{i} \leq \alpha$ is given as $\hat{\beta}_{i}^{\text {lasso }}=\operatorname{sign}\left(\hat{\beta}_{i}\right)\left[\left|\hat{\beta}_{i}\right|-\lambda\left(\alpha, \hat{\sigma}_{i}\right)\right]^{+}$.

500 Note that the bias correction depends on the p-value threshold $\alpha$ for including SNPs.

Maximum likelihood estimator to reduce winner's curse bias

502 Following Zhong and Prentice ${ }^{19}$, we assume $\hat{\beta}_{i} \sim N\left(\beta_{i}, \sigma_{i}^{2}\right)$ with $\sigma_{i}^{2}$ approximated by $\hat{\sigma}_{i}^{2}$. By 503 conditioning on $P_{i} \leq \alpha$ or equivalently $\left|\hat{\beta}_{i}\right| \geq \lambda, \hat{\beta}_{i}$ follows a truncated normal distribution with an explicit density function

$$
\frac{\frac{1}{\sigma_{i}} \phi\left(\left(\widehat{\beta}_{i}-\beta_{i}\right) / \sigma_{i}\right)}{\Phi\left(\beta_{i} / \sigma_{i}-\lambda / \sigma_{i}\right)+\Phi\left(-\beta_{i} / \sigma_{i}-\lambda / \sigma_{i}\right)} I\left(\left|\hat{\beta}_{i}\right| \geq \lambda\left(\alpha, \hat{\sigma}_{i}\right)\right) .
$$

506 We derived the estimator $\hat{\beta}_{i}^{m l e}$ by maximizing the conditional likelihood numerically using $\mathrm{R}$.

507 Again, $\hat{\beta}_{i}^{m l e}$ depends on the p-value threshold $\alpha$ for including SNPs. For computational 508 efficiency, we pre-calculated $\hat{\beta}_{i}^{\text {mle }}$ at a required precision for all predefined $\mathrm{p}$-value thresholds.

\section{Appendix B: Theoretical prediction performance assuming independent SNPs}

510 Suppose that for a given trait of interest $Y$, there are two predefined SNP sets: the high priority

511 (HP) SNP set $S_{1}$ and the low priority (LP) SNP set $S_{2}$. SNPs have been pruned and are in linkage 
512 equilibrium. We assume that $S_{1}$ has $M_{1}$ independent susceptibility SNPs and $M_{3}$ null SNPs while

$513 S_{2}$ has $M_{2}$ susceptibility SNPs and $M_{4}$ independent null SNPs. Following Chatterjee et al. ${ }^{11}$, we

514 assume that the true relationship between outcome $Y$ and independent susceptibility SNPs is

515 modeled as follows:

$$
Y=\sum_{i=1}^{M_{1}} \beta_{1 i} g_{1 i}+\sum_{j=1}^{M_{2}} \beta_{2 j} g_{2 j}+\sum_{k=1}^{M_{3}} 0 \cdot g_{3 k}+\sum_{l=1}^{M_{4}} 0 \cdot g_{4 l}+\epsilon
$$

516 where all $Y$ and the genotypic values $g$ 's are standardized so that $E(Y)=0, \operatorname{Var}(Y)=1$,

$517 E(g)=0$ and $\operatorname{Var}(g)=1$, and the error term $\epsilon \sim N\left(0, \sigma^{2}\right)$ and is independent of the genotypic 518 values.

519 From a discovery GWAS data set of size $N$, we have regression coefficient $\widehat{\beta}_{i}$ and two-sided p-

520 value $P_{i}$ for each SNP. We build an additive prediction model by including SNPs in $S_{1}$ with $P$ -

521 value $\leq \alpha_{1}$ and SNPs in $S_{2}$ with $P$-value $\leq \alpha_{2}:$

$$
\hat{Y}\left(\alpha_{1}, \alpha_{2}\right)=\sum_{i=1}^{M_{1}} \hat{\beta}_{1 i} \gamma_{1 i}\left(\alpha_{1}\right) g_{1 i}+\sum_{j=1}^{M_{2}} \hat{\beta}_{2 j} \gamma_{2 j}\left(\alpha_{2}\right) g_{2 j}+\sum_{k=1}^{M_{3}} \hat{\beta}_{3 k} \gamma_{3 k}\left(\alpha_{1}\right) g_{3 k}+\sum_{l=1}^{M_{4}} \hat{\beta}_{4 l} \gamma_{4 l}\left(\alpha_{2}\right) g_{4 l},
$$

522 where $\gamma(\alpha)=I(P \leq \alpha)$ with $I(\cdot)$ being an indicator function.

523 The predictive correlation coefficient (PCC) for the predictive model can be expressed as

$$
\begin{aligned}
& \operatorname{PCC}\left(\alpha_{1}, \alpha_{2}\right)=\operatorname{cor}\left(Y, \hat{Y}\left(\alpha_{1}, \alpha_{2}\right)\right) \\
& =\frac{\sum_{i=1}^{M_{1}} \beta_{1 i} \hat{\beta}_{1 i} \gamma_{1 i}\left(\alpha_{1}\right)+\sum_{j=1}^{M_{2}} \beta_{2 j} \hat{\beta}_{2 j} \gamma_{2 j}\left(\alpha_{2}\right)}{\sqrt{\sum_{i=1}^{M_{1}} \hat{\beta}_{1 i}^{2} \gamma_{1 i}\left(\alpha_{1}\right)+\sum_{j=1}^{M_{2}} \hat{\beta}_{2 j}^{2} \gamma_{2 j}\left(\alpha_{2}\right)+\sum_{k=1}^{M_{3}} \hat{\beta}_{3 k}^{2} \gamma_{3 k}\left(\alpha_{1}\right)+\sum_{l=1}^{M_{4}} \hat{\beta}_{4 l}^{2} \gamma_{4 l}\left(\alpha_{2}\right)}} .
\end{aligned}
$$


524 Following Chatterjee et al. (2014), one can verify that PCC follows a normal distribution by the

525 central limit theorem and the strong law of large numbers. Therefore, the expected value of PCC

526 can be approximated as

$E\left(P C C\left(\alpha_{1}, \alpha_{2}\right)\right)$

$$
\begin{aligned}
& =\frac{\sum_{i=1}^{M_{1}} \beta_{1 i} e_{N, \alpha_{1}}\left(\beta_{1 i}\right) \operatorname{pow}\left(N, \beta_{1 i}, \alpha_{1}\right)+\sum_{j=1}^{M_{2}} \beta_{2 j} e_{N, \alpha_{2}}\left(\beta_{2 j}\right) \operatorname{pow}\left(N, \beta_{2 j}, \alpha_{2}\right)}{\sqrt{\sum_{i=1}^{M_{1}} v_{N, \alpha_{1}}\left(\beta_{1 i}\right) \operatorname{pow}\left(N, \beta_{1 i}, \alpha_{1}\right)+\sum_{j=1}^{M_{2}} v_{N, \alpha_{2}}\left(\beta_{2 j}\right) \operatorname{pow}\left(N, \beta_{2 j}, \alpha_{2}\right)+M_{3} \alpha_{1} v_{N, \alpha_{1}}(0)+M_{4} \alpha_{2} v_{N, \alpha_{2}}(0)}}, \\
& \approx \frac{M_{1} \int \beta e_{N, \alpha_{1}}(\beta) \operatorname{pow}\left(N, \beta, \alpha_{1}\right) f_{1}(\beta) d \beta+M_{2} \int \beta e_{N, \alpha_{2}}(\beta) \operatorname{pow}\left(N, \beta, \alpha_{2}\right) f_{2}(\beta) d \beta}{\sqrt{M_{1} \int \beta v_{N, \alpha_{1}}(\beta) \operatorname{pow}\left(N, \beta, \alpha_{1}\right) f_{1}(\beta) d \beta+M_{2} \int \beta v_{N, \alpha_{2}}(\beta) \operatorname{pow}\left(N, \beta, \alpha_{2}\right) f_{2}(\beta) d \beta+M_{3} \alpha_{1} v_{N, \alpha_{1}}(0)+M_{4} \alpha_{2} v_{N, \alpha_{2}}(0)}}
\end{aligned}
$$

527 where $e_{N, \alpha}(\beta)=E(\hat{\beta} \mid \gamma(\alpha)=1), v_{N, \alpha}(\beta)=E\left(\hat{\beta}^{2} \mid \gamma(\alpha)=1\right), \operatorname{pow}(N, \beta, \alpha)$ is power to detect

528 a SNP with effect size $\beta$ at a significance level $\alpha$ in a GWAS with size $N$, and $f_{1}(\cdot)$ and $f_{2}(\cdot)$ are

529 effect-size distributions for HP and LP susceptibility SNPs, respectively.

530 In our numerical calculations, we assumed that the effect sizes of the susceptibility SNPs in the

531 HP and LP sets followed the same distribution $\beta \sim \pi N\left(0, \sigma_{1}^{2}\right)+(1-\pi) N\left(0, \sigma_{2}^{2}\right)$, consistent with

532 simulations. We performed grid search to identify the $\mathrm{p}$-value thresholds $\left(\alpha_{1}, \alpha_{2}\right)$ that maximizes

$533 E\left(P C C\left(\alpha_{1}, \alpha_{2}\right)\right)$. For binary disease outcomes, the area under the curve (AUC) can be expressed

534 as a function of PCC, as shown in Chatterjee et al. (2014).

535

536

537

538

539

540

541

542

543

544

545

\section{Acknowledgements}

This study utilized the high-performance computational capabilities of the Biowulf Linux cluster at the National Institutes of Health, Bethesda, MD. (http://biowulf.nih.gov). This study made use of data generated by the Wellcome Trust Case Control Consortium (WTCCC). A full list of the investigators who contributed to the generation of the data is available at www.wtccc.org.uk.

Funding for the WTCCC project was provided by the Wellcome Trust under award 076113. J.S. and N.C. were supported by the NIH intramural research program. The TRICL Consortium was supported by NIH grant U19 CA148127. We thank Hilary Kiyo Finucane and Alkes Price for providing the annotation data for conserved DNA regions. We would like to acknowledge all the investigators, their support staff, and their funding support who contributed to GWAS of lung 
546 cancer among non-smoking females in Asia, as part of the Female Lung Cancer Consortium in

547 Asia (FLCCA), described in reference 31. We would like to acknowledge all the investigators,

548 their support staff, and their funding support who contributed to GWAS of bladder cancer,

549 described in reference 28 and in reference 29.

550

551 Web Resources

552 The URLs for data provide herein are as follows:

553 Annotation for conserved genomic regions: http://compbio.mit.edu/human-constraint/data/gff/

554 DIAGRAM type 2 diabetes summary statistics, http://diagram-consortium.org/downloads.html

555 GERA GWAS data; http://www.ncbi.nlm.nih.gov/projects/gap/cgi-

556 bin/study.cgi?study id=phs000674.v1.p1

557 IMPUTE2, https://mathgen.stats.ox.ac.uk/impute/impute v2.html

558 Psychiatric Genomic Consortium (PGC2), schizophrenia summary statistics,

559 http://www.med.unc.edu/pgc/downloads

560 Histone mark and DHS peak data, http://www.roadmapproject.org/

561 Conserved genomic regions, http://compbio.mit.edu/human-constraint/data/gff/

562 Height, BMI, WC, WHP, obesity summary statistics from GIANT consortium,

563 http://www.broadinstitute.org/collaboration/giant/index.php/GIANT consortium

564 LDL, HDL, TC and triglycerides summary statistics,

565 http://www.broadinstitute.org/mpg/pubs/lipids2010/

566 eQTL and meQTL in adipos, http://www.muther.ac.uk/Data.html

567 Blood eQTL, http://genenetwork.nl/bloodeqtlbrowser/

568 SNAP, http://www.broadinstitute.org/mpg/snap/

569 Transdisciplinary Research In Cancer of the Lung (TRICL), http://u19tricl.org/

570 The code for PRS data analysis is available at http://dceg.cancer.gov/tools/analysis/functionalPRS

571

572 


\section{References}

606

607

608

609

610

611

612

613

614

615

616

617

1. Allen, H.L., Estrada, K., Lettre, G., Berndt, S.I., Weedon, M.N., Rivadeneira, F., Willer, C.J., Jackson, A.U., Vedantam, S., Raychaudhuri, S., et al. (2010). Hundreds of variants clustered in genomic loci and biological pathways affect human height. Nature 467, 832-838.

2. Wood, A.R., Esko, T., Yang, J., Vedantam, S., Pers, T.H., Gustafsson, S., Chun, A.Y., Estrada, K., Luan, J., Kutalik, Z., et al. (2014). Defining the role of common variation in the genomic and biological architecture of adult human height. Nature Genetics 46, 1173-1186.

3. Locke, A.E., Kahali, B., Berndt, S.I., Justice, A.E., Pers, T.H., Felix, R., Powell, C., Vedantam, S., Buchkovich, M.L., Yang, J., et al. (2015). Genetic studies of body mass index yield new insights for obesity biology. Nature 518, 197-U401.

4. Michailidou, K., Beesley, J., Lindstrom, S., Canisius, S., Dennis, J., Lush, M.J., Maranian, M.J., Bolla, M.K., Wang, Q., Shah, M., et al. (2015). Genome-wide association analysis of more than 120,000 individuals identifies 15 new susceptibility loci for breast cancer. Nature Genetics 47, 373-U127.

5. Al Olama, A.A., Kote-Jarai, Z., Berndt, S.I., Conti, D.V., Schumacher, F., Han, Y., Benlloch, S., Hazelett, D.J., Wang, Z.M., Saunders, E., et al. (2014). A meta-analysis of 87,040 individuals identifies 23 new susceptibility loci for prostate cancer. Nature Genetics 46, 1103-1109.

6. Mavaddat, N., Pharoah, P.D., Michailidou, K., Tyrer, J., Brook, M.N., Bolla, M.K., Wang, Q., Dennis, J., Dunning, A.M., Shah, M., et al. (2015). Prediction of breast cancer risk based on profiling with common genetic variants. J Natl Cancer Inst 107.

7. Yang, J., Benyamin, B., McEvoy, B.P., Gordon, S., Henders, A.K., Nyholt, D.R., Madden, P.A., Heath, A.C., Martin, N.G., Montgomery, G.W., et al. (2010). Common SNPs explain a large proportion of the heritability for human height. Nat Genet 42, 565-569.

8. Yang, J., Lee, S.H., Goddard, M.E., and Visscher, P.M. (2011). GCTA: a tool for genome-wide complex trait analysis. Am J Hum Genet 88, 76-82.

9. Park, J.H., Wacholder, S., Gail, M.H., Peters, U., Jacobs, K.B., Chanock, S.J., and Chatterjee, N. (2010). Estimation of effect size distribution from genome-wide association studies and implications for future discoveries. Nature Genetics 42, 570-U139.

10. Dudbridge, F. (2013). Power and predictive accuracy of polygenic risk scores. PLoS Genet 9, e1003348.

11. Chatterjee, N., Wheeler, B., Sampson, J., Hartge, P., Chanock, S.J., and Park, J.H. (2013). Projecting the performance of risk prediction based on polygenic analyses of genome-wide association studies. Nat Genet 45, 400-405, 405e401-403.

12. Stahl, E.A., Wegmann, D., Trynka, G., Gutierrez-Achury, J., Do, R., Voight, B.F., Kraft, P., Chen, R., Kallberg, H.J., Kurreeman, F.A., et al. (2012). Bayesian inference analyses of the polygenic architecture of rheumatoid arthritis. Nat Genet 44, 483-489.

13. Purcell, S.M., Wray, N.R., Stone, J.L., Visscher, P.M., O'Donovan, M.C., Sullivan, P.F., Sklar, P., Ruderfer, D.M., McQuillin, A., Morris, D.W., et al. (2009). Common polygenic variation contributes to risk of schizophrenia and bipolar disorder. Nature 460, 748-752.

14. International Schizophrenia, C., Purcell, S.M., Wray, N.R., Stone, J.L., Visscher, P.M., O'Donovan, M.C., Sullivan, P.F., and Sklar, P. (2009). Common polygenic variation contributes to risk of schizophrenia and bipolar disorder. Nature 460, 748-752.

15. Golan, D., and Rosset, S. (2014). Effective Genetic-Risk Prediction Using Mixed Models. Am J Hum Genet 95, 383-393.

16. Speed, D., and Balding, D.J. (2014). MultiBLUP: improved SNP-based prediction for complex traits. Genome Research 24, 1550-1557. 
618

619

620

621

622

623

624

625

626

627

628

629

630

631

632

633

634

635

636

637

638

639

640

641

642

643

644

645

646

647

648

649

650

651

652

653

654

655

656

657

658

659

660

661

662

663

17. Maier, R., Moser, G., Chen, G.B., Ripke, S., Coryell, W., Potash, J.B., Scheftner, W.A., Shi, J.X., Weissman, M.M., Hultman, C.M., et al. (2015). Joint Analysis of Psychiatric Disorders Increases Accuracy of Risk Prediction for Schizophrenia, Bipolar Disorder, and Major Depressive Disorder. American Journal of Human Genetics 96, 283-294.

18. Tibshirani, R. (1996). Regression shrinkage and selection via the Lasso. J Roy Stat Soc B Met 58, 267288.

19. Zhong, H., and Prentice, R.L. (2010). Correcting "winner's curse" in odds ratios from genomewide association findings for major complex human diseases. Genet Epidemiol 34, 78-91.

20. Schork, A.J., Thompson, W.K., Pham, P., Torkamani, A., Roddey, J.C., Sullivan, P.F., Kelsoe, J.R., O'Donovan, M.C., Furberg, H., Schork, N.J., et al. (2013). All SNPs Are Not Created Equal: Genome-Wide Association Studies Reveal a Consistent Pattern of Enrichment among Functionally Annotated SNPs. Plos Genetics 9.

21. Gusev, A., Lee, S.H., Trynka, G., Finucane, H., Vilhjalmsson, B.J., Xu, H., Zang, C., Ripke, S., BulikSullivan, B., Stahl, E., et al. (2014). Partitioning heritability of regulatory and cell-type-specific variants across 11 common diseases. Am J Hum Genet 95, 535-552.

22. Finucane, H.K., Bulik-Sullivan, B., Gusev, A., Trynka, G., Reshef, Y., Loh, P.R., Anttila, V., Xu, H., Zang, C., Farh, K., et al. (2015). Partitioning heritability by functional annotation using genome-wide association summary statistics. Nat Genet.

23. Purcell, S., Neale, B., Todd-Brown, K., Thomas, L., Ferreira, M.A., Bender, D., Maller, J., Sklar, P., de Bakker, P.I., Daly, M.J., et al. (2007). PLINK: a tool set for whole-genome association and population-based linkage analyses. Am J Hum Genet 81, 559-575.

24. Landi, M.T., Chatterjee, N., Yu, K., Goldin, L.R., Goldstein, A.M., Rotunno, M., Mirabello, L., Jacobs, K., Wheeler, W., Yeager, M., et al. (2009). A genome-wide association study of lung cancer identifies a region of chromosome 5 p15 associated with risk for adenocarcinoma. Am J Hum Genet 85, 679-691.

25. Lindblad-Toh, K., Garber, M., Zuk, O., Lin, M.F., Parker, B.J., Washietl, S., Kheradpour, P., Ernst, J., Jordan, G., Mauceli, E., et al. (2011). A high-resolution map of human evolutionary constraint using 29 mammals. Nature 478, 476-482.

26. (2007). Genome-wide association study of 14,000 cases of seven common diseases and 3,000 shared controls. Nature 447, 661-678.

27. Price, A.L., Patterson, N.J., Plenge, R.M., Weinblatt, M.E., Shadick, N.A., and Reich, D. (2006). Principal components analysis corrects for stratification in genome-wide association studies. Nat Genet 38, 904-909.

28. Rothman, N., Garcia-Closas, M., Chatterjee, N., Malats, N., Wu, X., Figueroa, J.D., Real, F.X., Van Den Berg, D., Matullo, G., Baris, D., et al. (2010). A multi-stage genome-wide association study of bladder cancer identifies multiple susceptibility loci. Nat Genet 42, 978-984.

29. Figueroa, J.D., Ye, Y., Siddiq, A., Garcia-Closas, M., Chatterjee, N., Prokunina-Olsson, L., Cortessis, V.K., Kooperberg, C., Cussenot, O., Benhamou, S., et al. (2014). Genome-wide association study identifies multiple loci associated with bladder cancer risk. Human Molecular Genetics 23, 13871398.

30. Wolpin, B.M., Rizzato, C., Kraft, P., Kooperberg, C., Petersen, G.M., Wang, Z.M., Arslan, A.A., BeaneFreeman, L., Bracci, P.M., Buring, J., et al. (2014). Genome-wide association study identifies multiple susceptibility loci for pancreatic cancer. Nature Genetics 46, 994-+.

31. Lan, Q., Hsiung, C.A., Matsuo, K., Hong, Y.C., Seow, A., Wang, Z.M., Hosgood, H.D., Chen, K.X., Wang, J.C., Chatterjee, N., et al. (2012). Genome-wide association analysis identifies new lung cancer susceptibility loci in never-smoking women in Asia. Nature Genetics 44, 1330-1335. 
664

665

666

667

668

669

670

671

672

673

674

675

676

677

678

679

680

681

682

683

684

685

686

687

688

689

690

691

692

693

694

695

696

697

698

699

700

701

702

703

704

705

706

707

708

709

32. Voight, B.F., Scott, L.J., Steinthorsdottir, V., Morris, A.P., Dina, C., Welch, R.P., Zeggini, E., Huth, C., Aulchenko, Y.S., Thorleifsson, G., et al. (2011). Twelve type 2 diabetes susceptibility loci identified through large-scale association analysis (vol 42, pg 579, 2010). Nature Genetics 43, 388-388.

33. Ripke, S., Neale, B.M., Corvin, A., Walters, J.T.R., Farh, K.H., Holmans, P.A., Lee, P., Bulik-Sullivan, B., Collier, D.A., Huang, H.L., et al. (2014). Biological insights from 108 schizophrenia-associated genetic loci. Nature 511, 421-+.

34. Shi, J.X., Levinson, D.F., Duan, J.B., Sanders, A.R., Zheng, Y.L., Pe'er, I., Dudbridge, F., Holmans, P.A., Whittemore, A.S., Mowry, B.J., et al. (2009). Common variants on chromosome 6p22.1 are associated with schizophrenia. Nature 460, 753-757.

35. Timofeeva, M.N., Hung, R.J., Rafnar, T., Christiani, D.C., Field, J.K., Bickeboller, H., Risch, A., McKay, J.D., Wang, Y., Dai, J., et al. (2012). Influence of common genetic variation on lung cancer risk: meta-analysis of 14900 cases and 29485 controls. Hum Mol Genet 21, 4980-4995.

36. Wang, Y.F., Mckay, J.D., Rafnar, T., Wang, Z.M., Timofeeva, M.N., Broderick, P., Zong, X.C., Laplana, M., Wei, Y.Y., Han, Y.H., et al. (2014). Rare variants of large effect in BRCA2 and CHEK2 affect risk of lung cancer. Nature Genetics 46, 736-741.

37. Peters, U., Jiao, S., Schumacher, F.R., Hutter, C.M., Aragaki, A.K., Baron, J.A., Berndt, S.I., Bezieau, S., Brenner, H., Butterbach, K., et al. (2013). Identification of Genetic Susceptibility Loci for Colorectal Tumors in a Genome-Wide Meta-analysis. Gastroenterology 144, 799-+.

38. Howie, B.N., Donnelly, P., and Marchini, J. (2009). A flexible and accurate genotype imputation method for the next generation of genome-wide association studies. PLoS Genet 5, e1000529.

39. Smoller, J.W., Craddock, N., Kendler, K., Lee, P.H., Neale, B.M., Nurnberger, J.I., Ripke, S., Santangelo, S., Sullivan, P.F., and Consortium, P.G. (2013). Identification of risk loci with shared effects on five major psychiatric disorders: a genome-wide analysis. Lancet 381, 1371-1379.

40. Lee, S.H., Ripke, S., Neale, B.M., Faraone, S.V., Purcell, S.M., Perlis, R.H., Mowry, B.J., Thapar, A., Goddard, M.E., Witte, J.S., et al. (2013). Genetic relationship between five psychiatric disorders estimated from genome-wide SNPs. Nature Genetics 45, 984-+.

41. Andreassen, O.A., Djurovic, S., Thompson, W.K., Schork, A.J., Kendler, K.S., O'Donovan, M.C., Rujescu, D., Werge, T., van de Bunt, M., Morris, A.P., et al. (2013). Improved Detection of Common Variants Associated with Schizophrenia by Leveraging Pleiotropy with CardiovascularDisease Risk Factors. American Journal of Human Genetics 92, 197-209.

42. Altshuler, D.M., Durbin, R.M., Abecasis, G.R., Bentley, D.R., Chakravarti, A., Clark, A.G., Donnelly, P., Eichler, E.E., Flicek, P., Gabriel, S.B., et al. (2012). An integrated map of genetic variation from 1,092 human genomes. Nature 491, 56-65.

43. Battle, A., Mostafavi, S., Zhu, X.W., Potash, J.B., Weissman, M.M., McCormick, C., Haudenschild, C.D., Beckman, K.B., Shi, J.X., Mei, R., et al. (2014). Characterizing the genetic basis of transcriptome diversity through RNA-sequencing of 922 individuals. Genome Research 24, 14-24.

44. Westra, H.J., Peters, M.J., Esko, T., Yaghootkar, H., Schurmann, C., Kettunen, J., Christiansen, M.W., Fairfax, B.P., Schramm, K., Powell, J.E., et al. (2013). Systematic identification of trans eQTLs as putative drivers of known disease associations. Nature Genetics 45, 1238-U1195.

45. Hao, K., Bosse, Y., Nickle, D.C., Pare, P.D., Postma, D.S., Laviolette, M., Sandford, A., Hackett, T.L., Daley, D., Hogg, J.C., et al. (2012). Lung eQTLs to Help Reveal the Molecular Underpinnings of Asthma. Plos Genetics 8.

46. Shi, J., Marconett, C.N., Duan, J., Hyland, P.L., Li, P., Wang, Z., Wheeler, W., Zhou, B., Campan, M., Lee, D.S., et al. (2014). Characterizing the genetic basis of methylome diversity in histologically normal human lung tissue. Nat Commun 5, 3365. 
47. Grundberg, E., Small, K.S., Hedman, A.K., Nica, A.C., Buil, A., Keildson, S., Bell, J.T., Yang, T.P., Meduri, E., Barrett, A., et al. (2012). Mapping cis- and trans-regulatory effects across multiple tissues in twins. Nat Genet 44, 1084-1089.

48. Grundberg, E., Meduri, E., Sandling, J.K., Hedman, A.K., Keildson, S., Buil, A., Busche, S., Yuan, W., Nisbet, J., Sekowska, M., et al. (2013). Global analysis of DNA methylation variation in adipose tissue from twins reveals links to disease-associated variants in distal regulatory elements. Am J Hum Genet 93, 876-890.

49. Hazelett, D.J., Rhie, S.K., Gaddis, M., Yan, C.L., Lakeland, D.L., Coetzee, S.G., Henderson, B.E., Noushmehr, H., Cozen, W., Kote-Jarai, Z., et al. (2014). Comprehensive Functional Annotation of 77 Prostate Cancer Risk Loci. Plos Genetics 10.

50. Speliotes, E.K., Willer, C.J., Berndt, S.I., Monda, K.L., Thorleifsson, G., Jackson, A.U., Allen, H.L., Lindgren, C.M., Luan, J., Magi, R., et al. (2010). Association analyses of 249,796 individuals reveal 18 new loci associated with body mass index. Nature Genetics 42, 937-U953.

51. Berndt, S.I., Gustafsson, S., Magi, R., Ganna, A., Wheeler, E., Feitosa, M.F., Justice, A.E., Monda, K.L., Croteau-Chonka, D.C., Day, F.R., et al. (2013). Genome-wide meta-analysis identifies 11 new loci for anthropometric traits and provides insights into genetic architecture. Nature Genetics 45 , 501-U569.

52. Teslovich, T.M., Musunuru, K., Smith, A.V., Edmondson, A.C., Stylianou, I.M., Koseki, M., Pirruccello, J.P., Ripatti, S., Chasman, D.I., Willer, C.J., et al. (2010). Biological, clinical and population relevance of 95 loci for blood lipids. Nature 466, 707-713.

53. Sampson, J.N., Wheeler, W.A., Yeager, M., Panagiotou, O., Wang, Z., Berndt, S.I., Lan, Q., Abnet, C.C., Amundadottir, L.T., Figueroa, J.D., et al. (2015). Analysis of Heritability and Shared Heritability Based on Genome-Wide Association Studies for Thirteen Cancer Types. J Natl Cancer Inst 107.

54. Vilhjalmsson, B.J., Yang, J., Finucane, H.K., Gusev, A., Lindstrom, S., Ripke, S., Genovese, G., Loh, P.R., Bhatia, G., Do, R., et al. (2015). Modeling Linkage Disequilibrium Increases Accuracy of Polygenic Risk Scores. Am J Hum Genet 97, 576-592.

55. Park, T., and Casella, G. (2008). The Bayesian Lasso. Journal of the American Statistical Association 103, 681-686.

56. Kilpinen, H., Waszak, S.M., Gschwind, A.R., Raghav, S.K., Witwicki, R.M., Orioli, A., Migliavacca, E., Wiederkehr, M., Gutierrez-Arcelus, M., Panousis, N.I., et al. (2013). Coordinated Effects of Sequence Variation on DNA Binding, Chromatin Structure, and Transcription. Science 342, 744747.

57. McVicker, G., van de Geijn, B., Degner, J.F., Cain, C.E., Banovich, N.E., Raj, A., Lewellen, N., Myrthil, M., Gilad, Y., and Pritchard, J.K. (2013). Identification of Genetic Variants That Affect Histone Modifications in Human Cells. Science 342, 747-749. 


\section{Figure legend}

\section{Figure 1. Theoretic investigation of prediction performance and optimal thresholds for SNP selection in 2D PRS.}

The theoretic calculation assumes $M=53,163$ independent SNP, of which 5,000 are causal for a binary trait, similar to simulation studies. The high-prior (HP) SNP set has 5,000 SNPs and the low-prior (LP) SNP set has 48,163 SNPs. $\triangle$ is the enrichment fold of HP SNPs in the causal SNP set. (A) The prediction AUC for 1D PRS and 2D PRS. (B) The optimal $P$-value thresholds for including HP and LP SNPs in 2D PRS. For both plots, $\mathrm{x}$-coordinate is the discovery sample size, assuming equal number of cases and controls.

Figure 2. Genetic risk prediction for type-2 diabetes. PRS models were built based on the summary statistics from a meta-analysis of DIAGRAM consortium and GERA data $(17,802$ cases and 105,109 controls in total) and validated in independent 1500 cases and 1500 controls in GERA. (A) Prediction $\mathrm{R}^{2}$ (observational scale) for 1D PRS with or without winner's curse correction. "NO": no winner's correction for association coefficients; "Lasso": regression coefficients were modified by a lasso-type correction; "MLE": association coefficients were modified by maximizing a likelihood function conditioning on selection. (B) Quantile-quantile plot for $-\log _{10}(P)$ for high priority (HP) SNPs v.s. low priority (LP) SNPs. SNPs were pruned to have pairwise $r^{2} \leq 0.1$. Here, the HP SNPs were eSNPs/meSNPs in adipose tissue or SNPs related with the $\mathrm{H} 3 \mathrm{~K} 4 \mathrm{me} 3$ mark in pancreatic islet cell line with data downloaded from the ROADMAP project. The HP SNPs were strongly enriched in the discovery data. (C) Prediction $\mathrm{R}^{2}$ for 2D PRS with lasso-type winner's curse correction. The SNP set was the same to (B). The best prediction $\left(\mathrm{R}^{2}=3.53 \%\right)$ was achieved when we included HP SNPs using criterion $P \leq 0.03$ and HP SNPs with $P \leq 0.005$. (D) The prediction $\mathrm{R}^{2}$, the area under the curve (AUC) and the significances for testing whether an alternative PRS was better than the standard 1D. The Nagelkerke $\mathrm{R}^{2}$ values were reported in Tables $\mathrm{S} 4$.

Figure 3. Comparison of polygenic risk prediction methods for 13 complex diseases. For all figures, the $\mathrm{y}$-coordinate is the prediction $\mathrm{R}^{2}$ in the observational scale. "1D" denotes 1D PRS; "2D, blood eSNPs" denotes 2D PRS using blood eSNPs as high-prior SNP set. In the x-axis, "NO" denotes PRS without winner's curse correction; "LASSO" and "MLE" denote lasso-type and MLE winner's curse correction, respectively. (A) Prediction $\mathrm{R}^{2}$ values for six diseases in WTCCC data, estimated based on five-fold cross-validation. (B) Prediction $\mathrm{R}^{2}$ values for three GWAS of cancers, estimated based on ten-fold cross-validation. (C) Prediction $\mathrm{R}^{2}$ values for four complex diseases estimated based on independent validation samples. 
781 Figure 4. Simulation results for comparing polygenic risk prediction methods and

782 different high priority SNP sets. Quantitative traits were simulated conditioning on the

783 genotypes of LD-pruned SNPs in lung cancer GWAS with 10,000 discovery samples and 1,924

784 validation samples. For each simulation, we used 5,000 causal SNPs and 9,940 high priority

785 (HP) SNPs (either randomly selected or the SNPs related with conserved regions). $\Delta$ denotes the 786 enrichment fold change of the HP SNP. In the x-axis, "1D" denotes 1D PRS without winner's

787 curse correction; "1D-LASSO(MLE)" denotes 1D PRS with lasso-type (MLE) correction; "2D-

788 random" indicates 2D PRS with HP SNP sets randomly selected from the LD-pruned SNPs in

789 the genome; "2D-CR” indicates 2D PRS using SNPs in conserved regions as HP SNPs. 

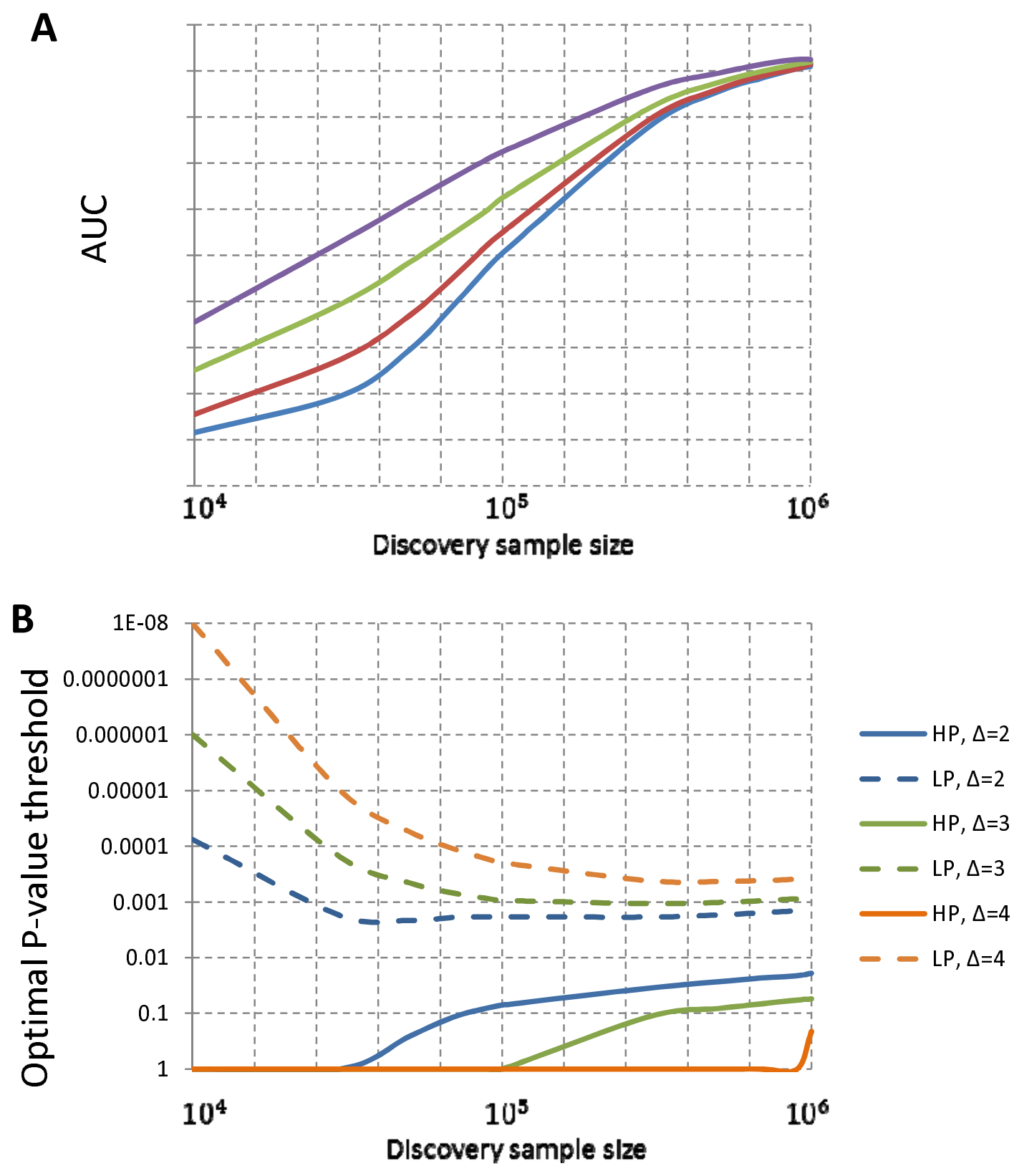

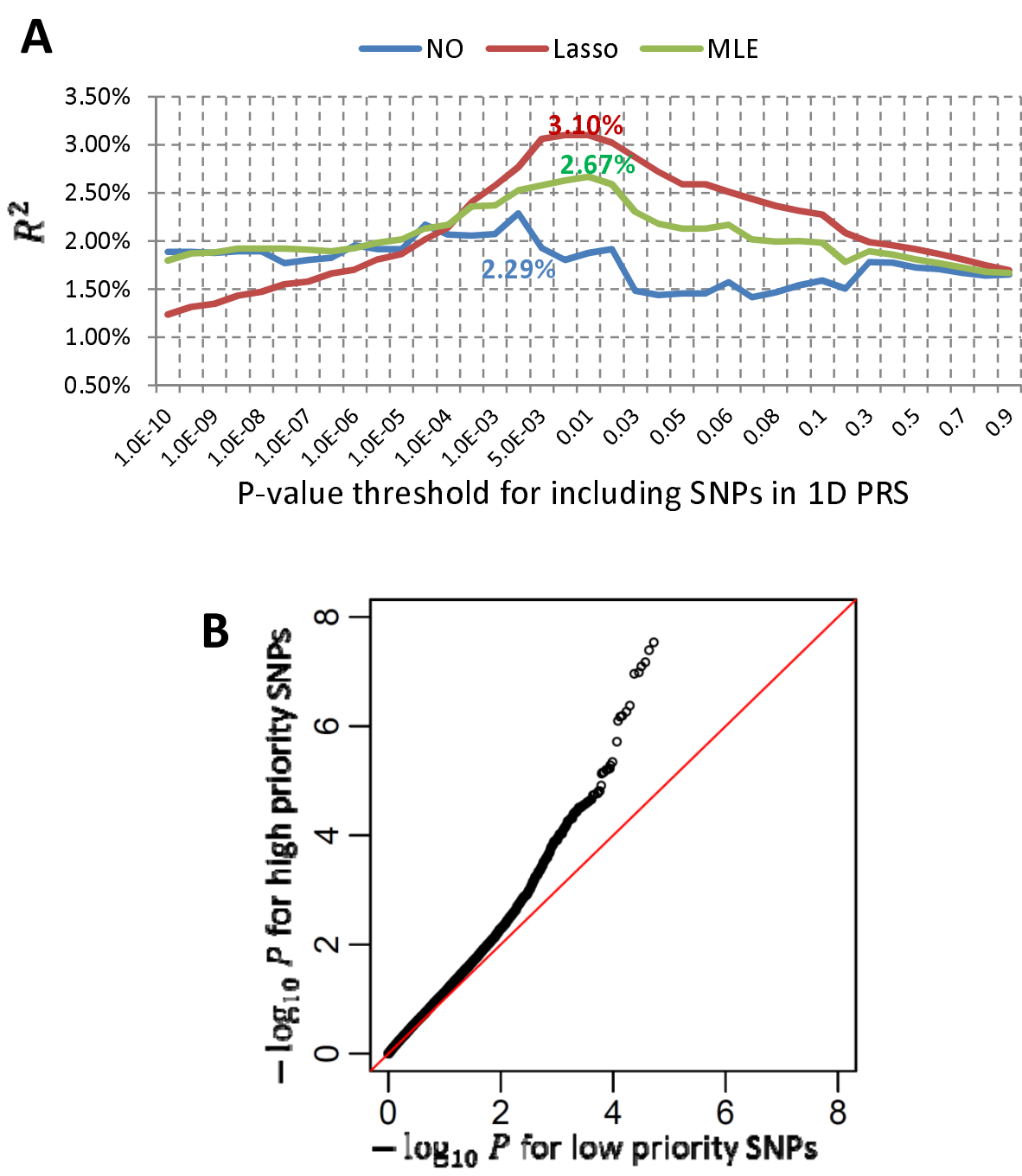
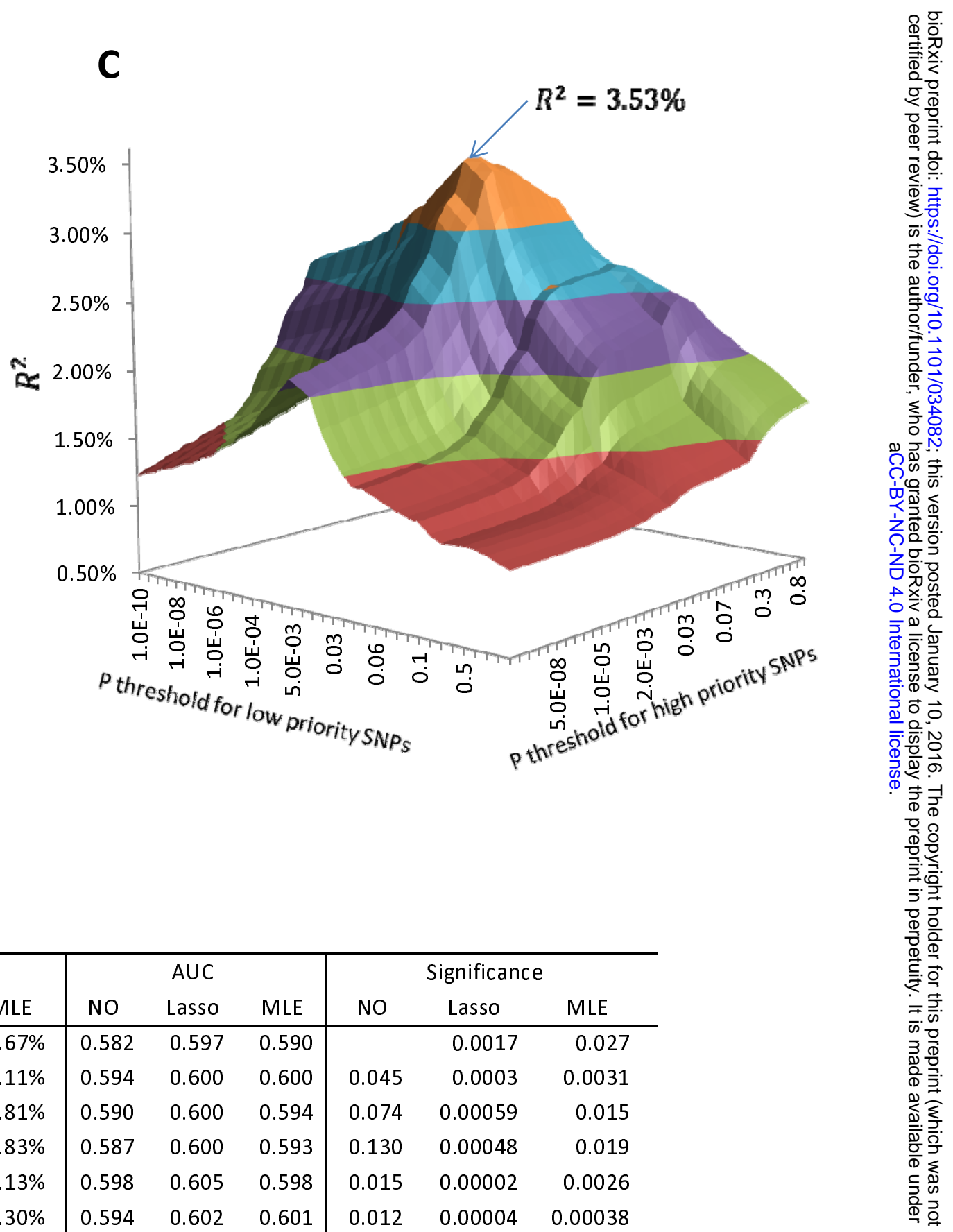

\begin{tabular}{|c|c|c|c|c|c|c|c|c|c|}
\hline \multirow{2}{*}{$\begin{array}{l}\text { PRS and high-priority } \\
\text { SNPs for 2D PRS }\end{array}$} & \multicolumn{3}{|c|}{ Prediction $\mathrm{R}^{2}$} & \multicolumn{3}{|c|}{ AUC } & \multicolumn{3}{|c|}{ Significance } \\
\hline & NO & Lasso & MLE & NO & Lasso & MLE & NO & Lasso & MLE \\
\hline 1D & $2.29 \%$ & $3.10 \%$ & $2.67 \%$ & 0.582 & 0.597 & 0.590 & & 0.0017 & 0.027 \\
\hline 2D, CR-SNPs & $2.73 \%$ & $3.32 \%$ & $3.11 \%$ & 0.594 & 0.600 & 0.600 & 0.045 & 0.0003 & 0.0031 \\
\hline 2D, histone SNPs, pancreatic islet & $2.58 \%$ & $3.23 \%$ & $2.81 \%$ & 0.590 & 0.600 & 0.594 & 0.074 & 0.00059 & 0.015 \\
\hline 2D, eSNPs/meSNPs & $2.58 \%$ & $3.28 \%$ & $2.83 \%$ & 0.587 & 0.600 & 0.593 & 0.130 & 0.00048 & 0.019 \\
\hline 2D, eSNPs/meSNPs and $\mathrm{H} 3 \mathrm{~K} 4 \mathrm{me} 3$ in islet & $2.90 \%$ & $3.53 \%$ & $3.13 \%$ & 0.598 & 0.605 & 0.598 & 0.015 & 0.00002 & 0.0026 \\
\hline 2D, eSNPs/meSNPs, CR-NPs & $2.92 \%$ & $3.48 \%$ & $3.30 \%$ & 0.594 & 0.602 & 0.601 & 0.012 & 0.00004 & 0.00038 \\
\hline
\end{tabular}


A
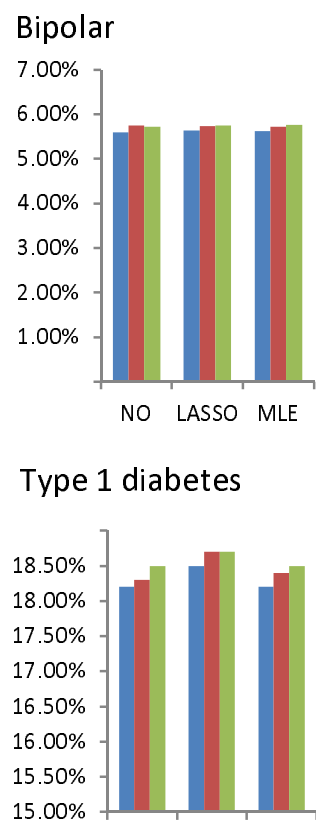

NO LASSO MLE

Hypertension

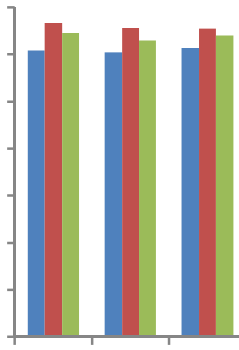

NO LASSO MLE

$=1 \mathrm{D}=2 \mathrm{D}$, blood eSNPS $=2 \mathrm{D}, \mathrm{CR}-\mathrm{SNPS}$

Rheumatoid
B
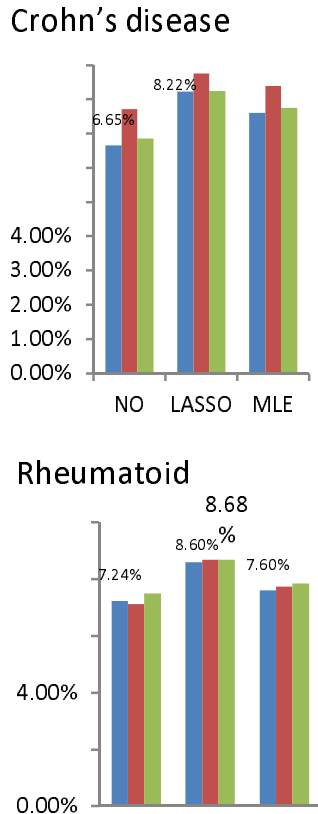

Coronary artery disease

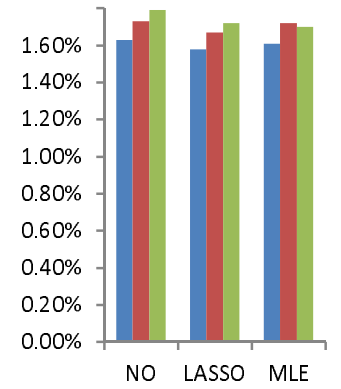

\section{Bladder cancer}

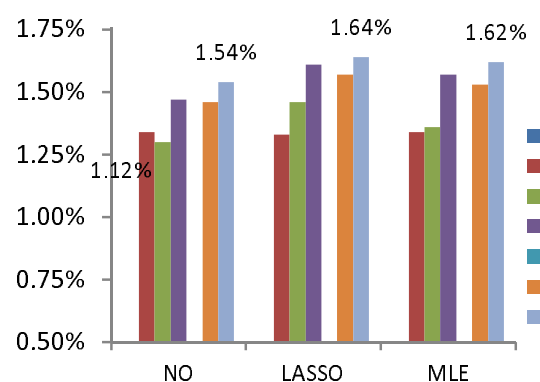

2D, CR-SNPs

2D, Blood-eSNPs

2D, H3K4me3, HAEC
2D, H3K9-14Ac, HAEC

$2 \mathrm{D}$, histone marks, bladder cell line

2D, functional SNPs in lung tissues

Asian non-smoking female lung cancer

$3.00 \% \quad 2.76 \% \quad 2.74 \% \quad 2.84 \%$

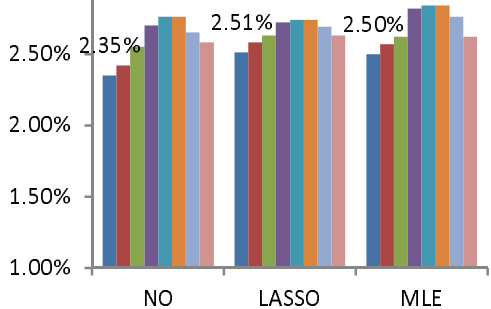

$=1 \mathrm{D}, \mathrm{D}, \mathrm{CR}-\mathrm{SNPS}$

2D, eSNPs/meSNPs in lung

2D, PT-0.01 SNPs

PT- 0.001 SNPs

D3K4me3 in HAEC

2D, Blood eSNPs

NO

MLE

\section{Pancreatic cancer}

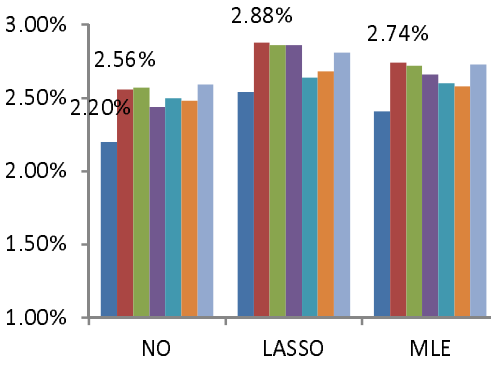

2D, CR-SNPs

2D, histone SNPs, pancreatic islet $2 \mathrm{D}$, histone SNPs, pancreatic

D, PT-0.001 SNPs

2D, PT-0.01 SNPs

2D, eSNPs/meSNPs in adipose
C

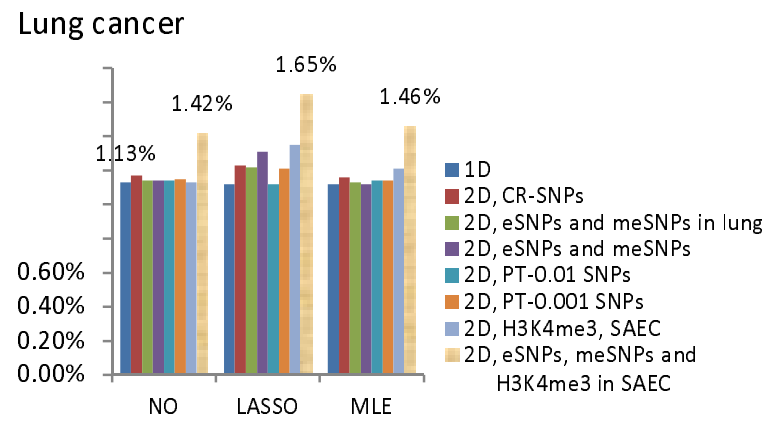

Schizophrenia

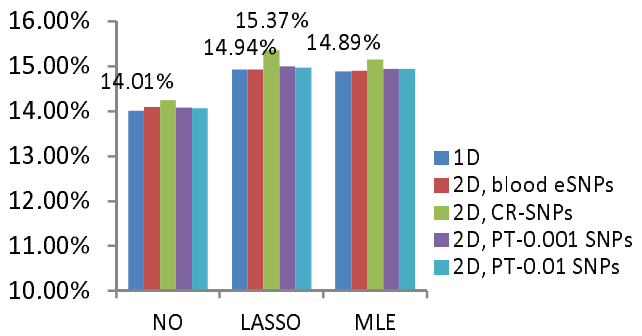

Colorectal cancer

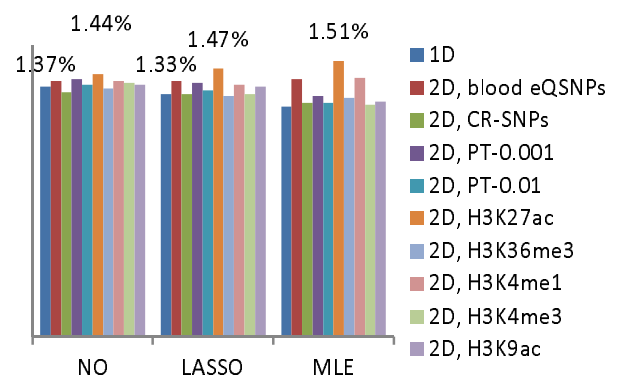

\section{Prostate cancer}

$8.00 \%$

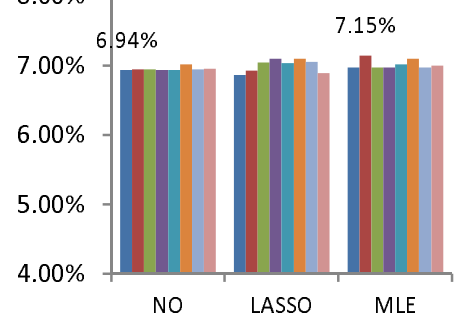

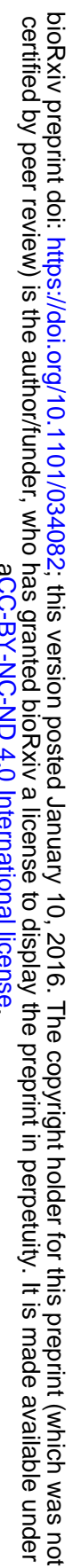

$2 \mathrm{D}$, blood eSNPs

2D, CR-SNPs

2D, PT-0.01

2D, H3K27Ac, -DHT 2D, H3K27Ac, +DHT 
Figure 4
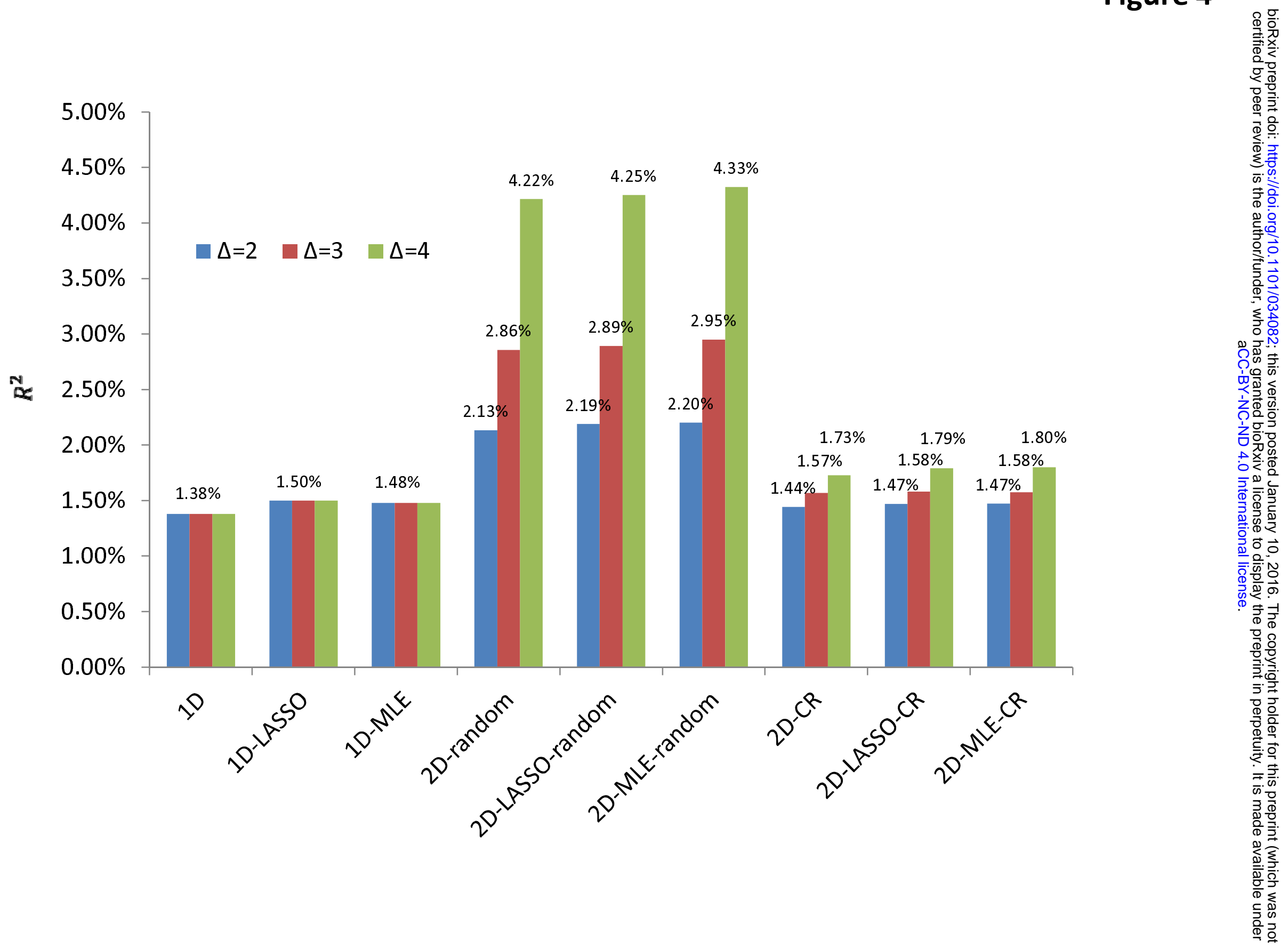